\title{
Errata for LBL-35369,
}

Garbesi, et al, Scale Dependence of Soil Permeability to Air: Measurement Method and Field Investigation, November 1995, and to be published in Water Resources Research

p. 12, second paragraph: replace "...range of driving-signal frequencies" with "...range of driving-signal periods"

p. 19, equation (25b): in denominator, replace "sin $\left(\delta_{1}\right) "$ with $" \sin \left(\theta_{1}\right) "$

p. 20, equation (25d): in denominator, replace "sin $\left(\delta_{2}\right)$ " with "sin $\left(\theta_{2}\right) "$

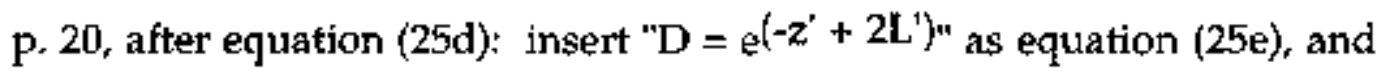

$$
\text { "E }=\mathrm{e}^{-\mathrm{Z}^{4}} \text { " as equation (250) }
$$

p. 22, fourth paragraph: insert in "To explore anisotropy, DDP measurements were made between the source..."

p. 23, first paragraph: insert in "To explore anisotropy, DDP measurements were made between the source..."

p. 24, fourth paragraph: change "Brimhal] et al. [1993] argue that..." to "Brimhall et al. [1993a] argue that..."; change "...at the Ben Lomond site [Brimhall et al., 1993]" to "...at the Ben Lomond site [Brimhall et al., 1993b]"; and insert "...and root tubules control dialational mixing by providing connected avenues..."

p. 32, definition for L': replace "Eq. 24b" with "Eq. 23b"

p. 33, definition for $z$ ": replace "Eq. 24a" with "Eq. 23a"

p. 34, Baehr and Hult (1989): replace with:

Baehr, A.L. and M.F. Hult, Determination of the air-phase permeability tensor of an unsaturated zone at the Bemidji, Minnesota, research site, in U.S. Geological Survey Toxic Substances Hydrology Program - Proceedings of the Techntcal Meeting, Phoenix, AZ, 1988, edited by G.E. Mallard and S.E. Ragone, pp. 55-65, Water Resources Investigations Rep. 88-4220, U.S. Geological Survey, Reston, VA, 1989.

p. 35, first Brimhall et al. reference: replace "1993" with "1993a"

p. 35, third Brimhall et al. reference: replace "1993" with "1993b"

P. 40, table footrote b: replace "Brimhall ef al., 1993" with "Brimhall and Lewis, 1992"

p. 46 , figure 4: the detector probe angle is $0^{\circ}$, not $90^{\circ}$

Jan. 19, 1996 



\section{DISCLAIMER}

This document was prepared as an account of work sponsored by the United States Government. While this document is believed to contain correct information, neither the United States Government nor any agency thereof, nor the Regents of the University of California, nor any of their employees, makes any warranty, express or implied, or assumes any legal responsibility for the accuracy, completeness, or usefulness of any information, apparatus, product, or process disclosed, or represents that its use would not infringe privately owned rights. Reference herein to any specific commercial product, process, or service by its trade name, trademark, manufacturer, or otherwise, does not necessarily constitute or imply its endorsement, recommendation, or favoring by the United States Government or any agency thereof, or the Regents of the University of California. The views and opinions of authors expressed herein do not necessarily state or reflect those of the United States Government or any agency thereof or the Regents of the University of California. 



\title{
Scale Dependence Of Soil Permeability To Air: Measurement Method And Field Investigation
}

\author{
Karina Garbesi ${ }^{*}$, Richard G. Sextro, Allen L. Robinson, John D. Wooley, Jonathan A. Owens, \\ And William W Nazaroff*** \\ Indoor Environment Program \\ Energy and Environment Division \\ Lawrence Berkeley National Laboratory, \\ Berkeley, California 94720 \\ * and Department of Geography and Environmental Studies \\ San José State University \\ San Joșé, Califơnnia 95192 \\ ** and Department of Civil Engineering \\ Universily of Califomia \\ Berkeley, California 94720
}

November 1995

This work was supported by the Kearney Foundation for Soil Science and by the Director, Office of Energy Research, Office of Health and Environmental Research, Environmental Sciences Division, and by the Assistant Secretary for Energy Efficiency and Renewable Energy. Office of Building Technologies of the U.S. Deparment of Energy (DOE), under Contract DE-ACO376SF00098. Additionel \$upport-for-K. Garbesi-was-provided by-the-Association- of Western Universities, the Switzer Foundation, and an Alexander Hollaender Postdoctoral Fellowship from DOE. Support for W. Nazaroff's effort was provided by National Science Foundation Grant BCS-9057298. 


\begin{abstract}
This work investigates the dependence soil air-permeability on sampling scale in nearsurface unsaturated soils. A new dual-probe dynamic pressure technique was developed to measure permeability in situ over different length scales and different spatial orientations in the soil. Soils at three sites were studied using the new technique. Each soil was found to have higher horizontal than vertical permeability. Significant scale dependence of permeability was also observed at each site. Permeability increased by a factor of 20 as sampling scale increased from 0.1 to $2 \mathrm{~m}$ in a sand soil vegetated with dry grass, and by a factor of 15 as sampling scale increased from 0.1 to $3.5 \mathrm{~m}$ in a sandy loam with mature Coast Live Oak trees (Quercus agrifolia). The results indicate that standard methods of permeability assessment can grossly underestimate advective transport of gas-phase contaminants through soils.
\end{abstract}




\section{INTRODUCTION}

Accurate assessment of soil permeability to air has important public heaith implications because advective transport of soil gas contaminants can result in elevated indoor concentrations of toxic and radjoactive pollutants. Indoor radon is an important example. Advective transport of soil-gas bearing radon is the dominant entry pathway into houses with higher than average radon concentrations [Nazaroff: 1992]. Radon decay products are known to cause lung cancer in humans [National Research Council, 1991]. There is also evidence of advective transport of volatile organic compounds from landfills, through unsaturated soils, and into nearby houses [Wood and Ponter, 1987; Califomia Air Resources Board. ]989; Hodgson et al., 1992; Little et al. 1992]. The California Air Resources Board found contaminants selected as indicators of hazardous waste in $70 \%$ of landfills tested, with similar constituents found in sites designated for hazardous or non-hazardous waste. Off site migration of landfill gas was detected at $83 \%$ of 273 landfills tested [Califomia Air Resources Board, 1989].

A number of studies have reported systematic underprediction of radon and soil-gas entry into buildings and test structures by mathematical mode]s [Garbesi and Sextro, 1989; Revzon et al., 1991; Andersen, 1992; Garbesi et al., 1993; Andersen ef al., 1995]. Nielson [1994] reported fairly good agreement between model predictions and entry rate; the model prediction of radon entry was within $20 \%$ of the measured value at carefully designed test structures and within a factor of 2.3 at real houses. Cripps [ 1993] also reported relatively good agreement between measured and modeled soil-gas entry into a test structure localed at a pit filled with sand. In both studies where modeled and measured values agreed reasonably wel] [Cripps, 1993; Nielson et al., 1994), the test structures and/or houses at which measurements were made were located on extremely disturbed uniform sands.

In the studies cited bere, the numerical models of radon transport have been validated against analytical solutions assuming homogeneous soils, indicating that the basic physics of the model is correct for a simple porous medium. All of the models use bulk soil permeability as input, some assume that soil may be considered homogeneous within regions [Garbesi et al., 1993] based on the model of Lourejo [ 1990], and some treat the soi] as anisotropic [Nielson et al, 1994] but minimally, regionally homogeneous [Garbesi, 1993]. Soil air-permeability is a critical input to the radon iransport models: air permeability tenos to control advective transport rates because the subsurface building shell typically imposes less resistance to flow than does the soil. 
A hypothesis put forward to explain the model underprediction of soil-gas and radon entry into a structure located in natural soil was that assessments of soil air-permeability, typically based on multiple small-scale measurements, underestimate permeability as 'seen' by the structure [Garbesi et al., 1993] If permeability increases with sampling length scale, then assessments of bulk soil permeability based on small-scale measurements will systematically underpredict advective transport rates for systems like houses or full-scale structures, which tend to interact with soils over considesably larger scales. Since it is the structure (heterogeneity) of the soil that can produce a scale effect, it is not surprising that studies carried out at disturbed-soil sites comprised of relatively uniform sand, would have better model-measurement consistency.

Here, we investigate the possibility that soil permeability to air depends upon sampling length scale. For some permeability measurement methods, the sampling length scale is obvious. For example, for measurements made on soil cores, the sampling scale is simply the core length. However, the sampling scale of a typical in-situ steady-pressure permeability measurement is not obvious. In these measurements, a steady flow of ait is introduced into (or withdrawn from) the soil via a probe inserted into the ground. This flow generaces a static pressure field in the soil surrounding the probe. The flow rate and the resulting disturbance pressure deternine the perneability via direct application of Darcy's law. (The term disturbance pressure is used henceforth to indicate the experimentally-induced deviation from the normal absolute pressure.) For many probe geometries the static pressure field can be approximated as spherical. For a spherical pressure source in an infinite homogeneous medium, the disturbance pressure diminishes inversely with distance from the source. Although the flow into the source, and hence the estimate of permeability, depends on the permeability of the entire soil block, the larger pressure gradient nearer the source calases the soil fiearer the probe to disproportionately influence the outcome of the measurement. Consequently, the sampling scale should be defined to incorporate the region over which most of the pressure gradient occurs. The larger the sampling surface is, the larger the sampling scale will be.

The sampiling scale (or effective sampling radius) of these steady-pressure permeability measurements has been defined as the distance at which the disturbance pressure field is $5 \%$ of its value at the probe's sampling tip [Fisk et al.. 1992]. In practice, this is taken to be the theoretically-determined distance at which the pressure field would be $5 \%$ of the probe-tip value in a homogeneous medium. That distance depends upon the size and geometry of the probe's sampling surface (e.g., a well screen or the open end of a blunt-end probe). Using this $5 \%$ criterion, and given the typical size of probes used for assessment of soil air permeability, the sampling scale of the steady-pressure measurements of soil air permeability are on the order of $10^{-1}$ 
m. For vapor extraction experiments (e.g., [Baehr et al., 1989; Massmann, 1989]), probes, and therefore also sampling scales, tend to be somewhat larger.

By the sarne criterion, houses, because of their large size, tend to interact with soils at a scale of - 3-10 m [Nazaroff et al., 1987; Garbesi and Sextro, 1989; Turk et al, 1990]. Therefore, if the soil permeability around a house is assessed using a technique that probes the soil at much smaller scale, and if the soit permeability increases significantly with sampling length scale, then the estimates of advective transport of contaminants into the house based on those measurements will significantly underestimate entry rates.

Although scale dependence of permeability is a recognized phenomenon in large-scale, water-saturated systems (e.g. [Bradbury and Muldoon, 1990; Wolf et al., 1991; Menduni, 1992; Celia and Rodrígues-Iturbe, 1993 j) it has been Jargely unstudied in unsaturated near-surface soils. In the literature we found only one such reference [Schery and Siegel, 1986]. Even in water-saturated systems, data are generally acquired using different methods at different scales, raising the question: Is observed scale dependence an artifact of systematic error in the sampling techniques or a property of the medium itse]f [Bradbury and Muldoon, 1990]?

The goal of this work was to make permeability measurements that bridge the gap between the scale at which in situ probes typically sample $\left(-10^{-1} \mathrm{~m}\right)$ and that at which houses interact with soils ( 1 to $10 \mathrm{~m}$ ); and to do so with minimal disruption to the site. We require a consistent metric of permeability at different length scaks. This is achieved by determining an equivalent homogeneous permeability at each length scale investigated. In addition, it is desirable to use the same measurement technique at different length scales, to eliminate the possibility of systematic error among techniques.

If a soil can be probed at the scale at which the system of interest operates, then, for many practical applications, the equivalent homogeneous permeability provides a sufficient and appropriate characterization of the potential for advective transport of gas-phase contaminants. Consider entry of soil-gas-bome contaminants into bouses: What is of interest is how the house 'sees' the soil (that is, the integrated behavior of the soil system), not the underlying complexities of the soil. Even if it were scientifically feasible to predict entry based on intensive small scale sampling and detailed modeling of the heterogeneous system (an issue that is debact in the literature), it is not fiscally feasible in most practical applications. To protect human bealth, site assessments must produce an accurate upper-limit assessment of exposure in a reasonable amnount 
of time given limited funds. In addition, measurements should result in minimal disnption of the sile.

To meet these goals, a new dual-probe dynamic pressure (DDP) measurement technique was developed to make measurements of equivalent homogeneous permeability in situ at different length scales. An intensive field investigation was carried out at one site to validate the DDP measurement technique and to investigate possible scale dependence of soil permeability. Strong scale dependence observed at that site prompted investigation of two very different soils at other sites to rule out the possibility that the effect was simply an anomalous characteristic of the first site. To validate the DDP technique its results were compared with single-probe steady pressure (SSP) measurements in sith. In addition, the in sith DDP measurements were replicated in a laboratory soil column to compare with steady-state measurements at known scale, and to verify that scale dependence observed in the field was not merely an antifact of the DDP measurement technique.

\section{DESIGN OF EXPERIMENTS}

\section{Dual-probe dynamic pressure apparatus}

Figure 1 is a schematic of the DDP measurement apparatus. A sinusoidal pressure oseillation is imposed at a source probe and is detected at a second probe. The time lag between the source and detector signals detemines the effective permeability of the path between the probes. By varying the distance between the source and detector probes measurements can be made over different length scales. Similarly, by varying the spatial orientation between the two probes, one can obtain information about anisotropy of soil permeability.

Several researchers have used the one-dimensional propagation of barometric pressure oscillations into the subsurface to characterize the vertical permeability of soil to air [Fukuda, 1955; Sherwood, 1974; Weeks, 1978; Schery and Siegel, 1986]. Narasimhan proposed using the propagation of earth tides through an aquifer to determine the specific storage to porosity ratio in the saturated medium [Narasimhan et al., 1984]. Black and Kipp [Black and Kipp, 1981] conducted a theoretical investigation of the possibility of using a system similar to the DDP technique for determining hydraulic diffusivity in aquifers and found it to be plausible under a limited set of conditions in that application. Unfortunately, their model of the experimental system assumed an infinite medium which will produce an invalid solution for the pressure signal in much of the soil domain in which we make measurements. In particular, the estimated sourcedetector phase shift (and hence the time lag) will be incorrect, resulting in an incorrect estimation of 
permeability. This fact can be seen plainly from the form of equation (16). The phase at the detector location depends upon the phases contributed by all of the real and images sources used to generate the effect of the boundary; this is particularly important near the soil surface or water tabie boundaries (see discussion below).

The advantage of a dynamic-pressure technique over steady-pressure methods, derives from the fact that the signal propagation speed, which is used as the indicator of perneability, is only weakly dependent on distance from the source ([Gorbesi, 1993], Chapter 3). This fact not only allows measurements to be made over relatively large length scales, but it gives similar weight to information coming from all points along the path. A resulting advantage of the DDP technique is that it is much less sensitive to the state of soil adjacent to the probe, which is likely to be disturbed during probe installation.

The DDP source probe consists of 15-cm length of cylindrical well screen welded into a pipe (15.4 $\mathrm{mm}$ ID, $3.1 \mathrm{~mm}$ wal]) fitted with a sealed and pointed end [Fisk et al., 1989; Fisk et at. 1992]. The size of the well-screen surface allows the pressure signal to propagate through many meters of soil without excessive attenuation while maintaining soil-gas velocities within the Darcy limit even at a distance of $1 \mathrm{~cm}$ from the source. The detector probes are generally a cut-off length of galvanized steel pipe $(8.5 \mathrm{~mm}$ ID, $2.7 \mathrm{~mm}$ wall), where the open end of the probe serves as the sampling surface. To prevent soil from entering the probe, a mandrel is inserted into the pipe during installation into an undersized, pre-drifled guide hole [Turk et al, 1987]. The soil at the opening, which can become packed during installation, is loosened by rotating a wood auger several $\mathrm{cm}$ into the soil through the probe. The top side of both probes are threaded and connected to flexible tubing with gas-tight fittings.

In the data-analysis model derived below, the source probe is approximated as a spherical source. An equivalent spherical radius is therefore determined for the well-screen probe. This approximation is reasonable as long as the aspect ratio (length/diameter) of the well screen is not too large and the screen length is small compared to the distances between the source and detector probes and between the source and the soil surface. The equivalent spherical radius is found by comparing a numerical simulation of a static pressure field around a cylindrical source with the analytical pretiction of the field surrounding a spherical source. In conducting this comparison, the size of the spherical source is adjusted until the far-field pressure is identical to that of a cylindrical source held at the same pressure. The equivalent spherical radius of the 15-cm well screen probe is $10 \mathrm{~cm}$. The maximum error resulting from this approximation is determined by geometric comparison of the cylindrical and spherical source geometries. The maximum distance between the surface of the well screen and the equivalent spherical source is $4 \mathrm{~cm}$. For a pressure 
signal propagating at constant velocity along a $1 \mathrm{~m}$ long path, this represents a maximum error in the estimated time lag of $0.04 / 1$, or $4 \%$. For our field measurements this corresponds with a maximum error in permeability of less than $2 \%$, which is much smaller than other uncertainties inherent in these kinds of measurements. The error is larger for shorter paths, but still small.

Commercially available data acquisition and control software was programmed to control the source signal and to simultaneously log the source and detector signals. The DDP source signal is created using two mass-flow controllers coupled to the soil probe by flexible tubing (Figure 1). To create a sinusoidally oscillating pressure signal centered about mean atmospheric pressure, one mass flow controller is computer driven to produce a sinusoidal flow with a positive offset $\left(\mathrm{Q}=\mathrm{q}_{1}+\mathrm{q}_{2} \sin (\omega \mathrm{t}), \mathrm{q}_{1} \geq \mathrm{q}_{2}\right)$, while the other is maintained at constant negative offset of amplitude $\mathrm{Q}=-\mathrm{q}_{1}$. The combination generates a pressure signal that oscillates about zero gauge pressure. Driving signal periods ranged from 30 to $120 \mathrm{~s}$. For low permeability soils, a lower driving frequency can be used to increase the amplitude of the detector signal. In our field tests, we found that it was sufficient to gather data for $\sim 20$ - 30 periods to obtain a good signal-to-noise ratio. The data logging rate is selected to produce a minimum of 2048 data points for Fourier analysis over 20 full cycles.

The pressure signals at the source and detector probes are measured using differential pressure transducers. Although we have used different models, we typically use detector-probe transducers with a $0-25 \mathrm{~Pa}$ operating range, having a $0.2 \mathrm{~Pa}$ resolution, and $0.05 \mathrm{~s}$ response time. The flow amplitude $\left(\mathrm{q}_{2}\right)$ of the source signal was adjusted to produce a pressure amplitude of $\sim 1 \mathrm{kPa}$. The transducer was sized accordingly. The source and detector signals are logged simultaneously and time stamped by an automated data acquisition system. Before the start of an experiment, the sinusoidally-oscillating source flow is vented to atmosphere and the source and detector probes are open to atmosphere to ensure neutral pressure. To begin an experiment, the source signal is switched to the source probe and all transducers are switched to the probes. These steps occur simultaneously using computer-controlled solenoid valves.

The non-probe sides of all pressure transducers are connected together and to a reference probe located far $(\sim 30 \mathrm{~m})$ from the cluster of source and detector probes. The purpose is to use the soil to filter noise from wind on the transducer reference ports. If this is not done, even modest winds can overwhelm the small $(\sim 1 \mathrm{~Pa})$ signals observed at the detector locations. This configuration allows very clear signals to be obtained even over the largest source-detector separation distances tested in the field $(4.5 \mathrm{~m})$. 
To ensure that artifficial time lags are not generated by differential response times of the pressure transducers, all transducers are calibrated to a common pressure source in the field before and after permeability data are collected. Time-lag data are corrected accordingly. The system curently in use is sensitive to differences in response times of $\sim 2 \mathrm{~ms}$. Differences in response times due to tubing lengths are calculated from the speed of sound in air. Accordingly, if the difference in tubing lengths for all probes is less than $30 \mathrm{~cm}$, then differences in response times from this factor are less than $1 \mathrm{~ms}$.

\section{Soil-column experiments}

DDP Gield experiments were reproduced in a laboratory soil column in one-dimensional form to ensure that scale dependence of permeability observed in the field was not an artifact of the DDP measurement technique. Experiments were conducted in homogeneously packed dry sand, which should have constant permeability regardless of length scale. DDP-like measurements were made over the same range of length scales over which scale dependence had been observed in natural soils in situ. As in the field experiments, the DDP source signal amplitude was $-1 \mathrm{kPa}$. Pressures were monitored using two electronic micromanometers and were logged with an automated data acquisition and control system. For comparison, steady-pressure permeability measurements were made at the same length scales. The column was used to perform a null-test. That is, dyramic measurements of permeability were made over different well-defined length scales in the column, in soil known to have relatively constant permeability (i.e., no scale dependence).

Shown in Figure 2, the 2.5-m long (0.15 $\mathrm{m}$ ID) soil-column was constructed of PVC pipe. The column has a sealed bottom cap to create a head space for the introduction of pressure signals and an optional cap for sealing the top. The soil is supported by a perforated aluminum plate covered with stainless steel mesh (100-mesh). Pressures are measured at 5 sampling ports ( $\mathrm{PO}$ to P5, Figure 2). P0, used to monitor the steady or dynamic source signal, is equivalent to the DDP source probe. $\mathrm{P1}-\mathrm{P} 4$, used for monitoring pressures at different distances from the source $(0.5$, $1.0,1.5$, and $1.85 \mathrm{~m}$, respectively), are equivalent to the DDP detector probes. The inside of each of the four ports is bored to accommodate $0.64 \mathrm{~cm} O D\left(1 / 4^{\prime \prime}\right)$ stainless steel tubing inserted so that pressure monitoring occurs atong the center line of the column. To facilitate uniform soil packing, the tubes were installed after the column was packed. The column was filled as uniformly as

possible to a height of $2.27 \mathrm{~m}$ with air-dried, sieved, and mixed, 60-mesh sand (particle grain size $\leq 0.25 \mathrm{~mm}$ ). The air filled porosity of the sand, $\varepsilon$, was $0.43 \pm 0.02$ [Garbesi, 1993]. 


\section{THEORY}

In this section we develop the closed-form mathematical models used to interpret the DDP field measurements, the DDP soil column measurements, and the steady-pressure measurements in the field and the soil column. All of the models use the following assumptions: the soil is homogeneous, isotropic, and isothermal; soil-water is unaffected by the measurements; the soil-gas behaves as an ideal gas; soil-gas transport is governed by Darcy's Law; slip flow can be neglected. The justification for a homogeneous soil model was discussed in the introduction, other assumptions are discussed below.

Although the model assumes an isotropic soil, anisotropy is readily detected from DDP measurements made from the same source probe to detector probes located in different directions (but the same distance) from the source. In fact, the relative lag times should give a good measure of the relative permeability if measurements are made at the same sampling length scale.

However, only a first order estimate of the absolute permeability can be obtained for anisotropic soils using the isotropic model described herein. If measurements indicate that a soil is strongly anisotropic, absolute directional permeabilities could be obtained by explicitly including the measured relative anisotropy in numerical soil model. This exercise is, however, beyond the scope of the current paper. Others have included soil layers, which can generate effective anisotropies, in closed-form models of steady-flow measurements [Baehr and Hult, 1991; Baehr and Joss, 1995] and one-dimensional dynamic measurements [Weeks, 1978].

The data analysis model treats the soil as isothermal, thus thermal convective fluxes are neglected. Natural convective fluxes from thermal gradients should oscillate over time scales (diurnal and seasonal) that are long compared to the source signal period. Since the Fourier analysis only utilizes signals at the source-signal frequency, the DDP technique- unlike staticpressure techniques-is insensitive thermal effects either in the environment or in the sampling lines. This fact also makes the system insensitive to barometric pressure fluctuations as long as the power at the experimental driving-signal frequency is low. The isothermal assumption also implies constant soil-gas viscosity. Since viscosity depends upon the square root of the absolute temperature [Noggle, 1985], it is relatively insensitive to environmental temperature variability.

Slip flow - also known as the Klinkenberg effect-is also neglected. This phenomenon describes the enhancement of flow due to non-zero velocities occurring at the grain surfaces. It becomes important when the pore size is on the order of the mean free path of the gas molecules $(\sim 0.065 \mu \mathrm{m}$ for air at standard temperature and pressure $)$. Slip flow increases as pore size and air pressure decrease. For the conditions of the DDP experiments, in which the source pressure 
signal is maintained within $1 \%$ of atmospheric, even for silt soils, the maximum tror from neglecting slip flow is less than $1 \%$ [Baehr and Hult, 1991].

The model assumes that soil water is unperturbed by the experiment. Thus, the soil is considered as a two-phase medium with gas as the mobile phase and solids plus water as the immobile phase. Since DDP rneasurements are made in soils with water content at or below field capacity (i.e., well drained soils), the matrix potential of soil water at field capacity defines the suction pressure (or capillary holding capacity) of the soil matrix on the water. Only pressure disturbances greater than this could significantly disturb soil water. Since matrix potential at field capacity is $10-30 \mathrm{kPa}$, the $\leq 1 \mathrm{kPa}$ disturbance pressures used in the DDP and SSP measurements will not significantly disturb the soil-water configuration [Hillel, 1980]. This is verified by field observations, which show no systematic changes in observed penmeability using either "blowing" or "sucking" static measurements, or given repeated DDP measurements.

The model uses Darcy's law to describe air flow through the soil. Darcy's law is valid for the two-phase medium described above, provided that the Reynolds number is less than -1 ([Bear, 1972], pg. 126). Darcy's law is defined, strictly speaking, for steady flow conditions. However, it is standard to use it to model non-steady flow. This approach is particularly reasonable bere given the long source-signal oscillation periods.

Combining Darcy's law and the continuity equation, and considering soil air as an incompressible ideal gas, Massmann [ 1989] shows that the equation for propagation of the pressure signal through the soil is given by

$$
\frac{\partial P}{\partial t}=\frac{k}{\varepsilon \mu} \nabla(P \nabla P)
$$

All variab]es and constants are defined in the notation section. Massmann [ 1989] shows that for small deviations from atmospheric pressure $(<1 \%)$, (1) can be approximated with negligible error by the linear transient diffusion equation:

$$
\frac{\partial \mathrm{P}}{\partial \mathrm{t}}=\frac{\mathrm{kP}_{\mathrm{a}}}{\varepsilon \mu} \nabla^{2} \mathrm{P} .
$$


Less restrictive simplifications of (1) have been examined [Muskat and Bostet, 1931; Baehr and Hult, 1989; Massmann, 1989]. However, for the conditions of these experiments, they are unnecessary. From (2) the pressure diffusivity of the system is defined as

$$
\mathrm{D}_{\mathrm{p}}=\mathrm{kP}_{\mathrm{a}} /(\varepsilon \mu) \text {. }
$$

Because the DDP measurement method, the permeability estimate is based on the diffusion of the pressure signal through the soil (2), the rate of which is determined by the diffusivity term (3), one must first obtain an accurate estimate of the air-filled porosity of the bulk soil ( $\varepsilon$ ). The average atmospheric pressure $\left(\mathrm{P}_{\mathrm{a}}\right)$ and the viscosity may be taken as constant (discussed below). In keeping with the concept of obtaining an equivalent homogeneous permeability over a given scale, the air-filled porosity must reflect the average property of the bulk medium as well. Therefore, if the soil of interest contains many rocks, and if porosity is determined from rock-free soil samples, the fractional volume of rock must be subtracted from the sample-determined porosity.

The researcher must also be sure that the frequency selected for the DDP experiments is sufficiently low that the signal fully probes the entire porosity of the soil. That is, if too high a frequency is selected, the signal will not have time to penetrate low permeability regions if their size is larger than the order of $\sqrt{\mathrm{D}_{\mathrm{p}} \mathrm{P}_{\mathrm{r}} / 2}$. In practice, this is easily checked by running experiments over a range of driving-signal frequencies ( 30 to $120 \mathrm{~s}$ ) and checking that the estimates of permeability remain constant. Since, porosity is entered into equation (3) at its constant, field-determined value, if the experimental signal is in fact probing progressively more of the soil porosity as the frequency is reduced, it will appear that the permeability is changing. Ideally, the lower the frequency the better, since the quasi-static conditions guarantee that the estimated permeability will converge on the steady-state Darcy flow equivalent. On the other hand, in practice it is desirable to limit the required sampling time. In our experience in the field, it was never necessary to run experiments with periods greater than $\sim 90 \mathrm{~s}$.

Model derivation for in situ DDP measurements :

We model the soil as having a constant pressure boundary at the soil surface and a no-flow boundary at the water table (or bedrock). Given the assumptions above, the solution for the soilgas pressure signal at any location may be found by first obtaining the solution to (2) for a sinusoidally oscillating source in an infinite medium (the IM solution), and then using the method 
of images to satisfy the two boundary conditions (see for example, [Reitz et al, 1979]). Briefly, to create a planar constant-pressure boundary, an image source of equal magnitude but opposite sign is placed equidistant from the boundary on the opposite side, such that the boundary bisects a line between the real and image sources. A planar no-flow boundary is created in a similar fashion using an image source of the same sign as the real source. Two solarces are required to satisfy one boundary; theoretically, an infinite number of images are required to satisfy two boundaries. The solution at any detector location is obtained by summing the $\mathrm{IM}$ solutions from all of the jmages, given the distance from each of the images at that location. Figure 3 shows the geometry and signs of the real source and the first few image sources.

A closed-form solution for the IM problem is obtained by solving only for the steadily oscillating part of the solution (i.e., by neglecting the transient par1). The time required to achieve this is on the order of $\mathrm{r}^{2} / \mathrm{D}_{\mathrm{p}}$. The full transient solution, obtained using Green's function and numerical integration, is given in [Garbesi, 1993].

If the pressure source is assumed to be spherical of radius b, then the IM problem can be solved by rewriting (2) in spherical coordinates

$$
\frac{\partial \mathrm{P}}{\partial \mathrm{t}}=\mathrm{D}_{\mathrm{p}} \frac{1}{\mathrm{r}^{2}} \frac{\partial}{\partial \mathrm{r}}\left(\mathrm{r}^{2} \frac{\partial \mathrm{P}}{\partial \mathrm{r}}\right)
$$

using the following boundary conditions:

$$
\begin{array}{ll}
P(b, t)=P_{0} \sin (\omega t) & \text { for } 1>0 \\
P(r \rightarrow \infty, t)=0 & \text { for } t>0
\end{array}
$$

Note that the initial condition is not needed because we are not solving for the transient part. This problem is readily solved using the method of complex combination [Myers, 1987] and the following transformation: $W(\mathrm{~L}, \mathrm{t})=\mathrm{r} \mathrm{P}(\mathrm{c}, \mathrm{t})[$ [Carslaw and Jaeger, 1959]. The timedependent pressure at a distance $r$ from the source is 


$$
\mathrm{P}(\mathrm{r}, \mathrm{t})=\mathrm{bP}_{\mathrm{o}} \frac{\exp (-\lambda(\mathrm{r}-\mathrm{b}) / \sqrt{2})}{\mathrm{r}} \sin \left(\omega t-\frac{\lambda(\mathrm{r}-\mathrm{b})}{\sqrt{2}}\right)
$$

The boundary conditions are then satisfied by adding the IM solutions (6) for the real source and an infinite number of image sources, each exerting an influence according to the its distance from the detector $\left(\mathrm{r}_{\mathrm{j}}\right)$. Therefore, the full solution is obtained from

$$
P_{d e t}(t)=\sum_{j=-\infty}^{\infty} P_{j}\left(r_{j}, t\right)
$$

where the distance from the jth source to the detector probe is given by

$$
r_{j}=\sqrt{h^{2}+\left[d+s\left(j-\frac{1+\cos (j \pi)}{2}\right)+w\left(j-\frac{1-\cos (j \pi)}{2}\right)\right]^{2}}
$$

As shown in Figure 3, $\mathrm{j}=0$ represents the real source, $\mathrm{j}=1$ to positive infinity represent the above-ground-surface image sources, and $\mathrm{j}=-1$ to negative infinity represent the below-groundsurface image sources.

From equations (6) and (8) and Figure 3, the disturbance pressure at the detector location due to the jth image source is

$$
P_{j}(t)=s_{j} A_{j} \sin \left(\omega t-\phi_{j}\right)
$$

where $\mathrm{s}_{\mathrm{j}}$ is the sign of the $\mathrm{jth}$ image 


$$
\mathrm{s}_{\mathrm{j}}=\sqrt{2} \cos \left(\mathrm{j} \frac{\pi}{2}+\frac{\pi}{4}\right) \text {, }
$$

$\mathrm{A}_{\mathrm{j}}$ is the amplitude of the pressure signal at the detector caused by the $\mathrm{jth}$ image

$$
A_{j}=b_{0} \frac{\exp \left(-\lambda\left(r_{j}-b\right) / \sqrt{2}\right)}{r_{j}} \text {, }
$$

and $\phi_{j}$ is the phase lag of the pressure signal at the detector caused by the jth image

$$
\phi_{\mathrm{j}}=\lambda\left(\mathrm{r}_{\mathrm{j}}-\mathrm{b}\right) / \sqrt{2}
$$

Combining (7) and (9), and the trigonometric identity for the sine of a difference of angles, the soil-gas pressure field at the location of the detector probe is

$$
P_{\operatorname{det}}(t)=\sin (\omega t) \sum_{j=-\infty}^{\infty} s_{j} A_{j} \cos \left(\phi_{j}\right)-\cos (\omega t) \sum_{j=-\infty}^{\infty} s_{j} A_{j} \sin \left(\phi_{j}\right) .
$$

Equation (13) can be simplified to the following form:

$$
P_{\operatorname{det}}(t)=\gamma \sin (\omega t-\beta)
$$

by defining the parameters $\gamma$ and $\beta$, such that following pair of equations hold true simultaneously: 


$$
\begin{aligned}
& \gamma \cos (\beta)=\sum_{j=-\infty}^{\infty} s_{j} A_{j} \cos \left(\phi_{j}\right), \\
& \gamma \sin (\beta)=\sum_{j=-\infty}^{\infty} s_{j} A_{j} \sin \left(\phi_{j}\right) .
\end{aligned}
$$

The phase lag between the source and the detector signals $(\beta)$ is found by solving (15a) and (15b) for $\beta$.

$$
\beta=\tan ^{-1}\left(\frac{\sum_{j=-\infty}^{\infty} s_{j} A_{j} \sin \left(\phi_{j}\right)}{\sum_{j=-\infty}^{\infty} s_{j} A_{j} \cos \left(\phi_{j}\right)}\right)
$$

From (14), the time lag for the signal to travel from the source to the detector location is

$$
T=\beta / \omega
$$

Theoretically an infinite number of image sources must be summed to match the boundary conditions. But the amplitude of the contribution of each image to the soil-gas pressure at the detector probe $\left(\mathrm{A}_{\mathrm{j}}\right)$ diminishes exponentially as the distance between the image source and the detector increases. Truncating the infinite series (equations $15 \mathrm{a}$ and $15 \mathrm{~b}$ ) to consider only images with $\lambda r_{j} / \sqrt{2} \leq 10$, yields results accurate to several significant figures-accuracy greater than the field measurements of time lag. The following conditions make more images necessary: more permeable soils, experiments with long driving-signal periods, a water table closer to the source probe. Generally less than 10 images are required. If the distance between the source probe and the water table is more than $10 \mathrm{~m}$ one frequently need only consider the real source and the first image source. In that case, the time lag for the signal to travel from the source to the detector location is 


$$
T=\frac{1}{\omega} \tan ^{-\mathrm{I}}\left(\frac{\mathrm{A}_{0} \sin \left(\phi_{0}\right)-\mathrm{A}_{1} \sin \left(\phi_{1}\right)}{\mathrm{A}_{0} \cos \left(\phi_{0}\right)-\mathrm{A}_{1} \cos \left(\phi_{1}\right)}\right) .
$$

One must be careful when computing the time lag from (17) or (18) because it is not uniquely determined by those equations [Chatfield, 1989]. Because the tangent function is only uniquely defined between $\pm \pi / 2$, as $\beta$ ( $\omega T$ T) in actuality exceeds $\pi / 2$, the output of $(16)$ is a negative angle equal to $\beta$ (actual) - $\pi$. Therefore, to obtain the conrect answer, one begins by calculating the time lag between the source and detector at a very low frequency (for which the time lag is guaranteed to be less than one quarter period). The frequency is then slowly increased, keeping track of the sign changes in (17), until the frequency matches that of the actual driving signal. The number of sign changes indicates the number of half periods that must be added to (17) to obtain the correct time lag.

A first order estimate of the anisotropy in permeability can be obtained using the isotropic model and the time lags measured from a single source probe to different detectors located the same distance from the soutce but at different spatial orientations in the soil, as illustrated in Figure 4. The data are interpreted using the concept of a hyduaulic conductivity ellipse ([Freeze and Cherry, 1979], p. 174). Assuming the principle axes of anisotropy align in the horizontal and vertical directions (i.e., $\mathrm{k}_{\mathrm{x}}=\mathrm{k}_{\mathrm{y}}=\mathrm{k}_{\mathrm{h}} \neq \mathrm{k}_{\mathrm{z}}=\mathrm{k}_{\mathrm{v}}$ ), the ellipse is given by

$$
\mathrm{x}^{2} / \mathrm{k}_{\mathrm{h}}+\mathrm{z}^{2} / \mathrm{k}_{\mathrm{v}}=1
$$

Equation (19) defines the relationship between permeability in the horizontal and the vertical directions. The permeability at any other orientation (defined by the angle $\theta$ from the horizontal as in Figure 4) is determined by the square root of the length of the vector from the origin to the ellipse. Thus, measurements made in two independent directions can yield approximate estimates of $k_{h}$ and $k_{v}$. 
Model derivation of DDP measurements in the soil-column:

The model is based on the steady solution of (2) for a sinusoidally oscillating pressure signal propagating one-dimensionally in a soil column. For this geometry, equation (2) simplifies to

$$
\frac{\partial \mathrm{P}}{\partial \mathrm{t}}=\mathrm{D}_{\mathrm{p}} \frac{\partial^{2} \mathrm{P}}{\partial \mathrm{z}^{2}},
$$

with the following boundary conditions:

$$
\begin{array}{ll}
P(z=0, t)=P_{0} \cos (\omega t) & \text { for } t>0 \\
P(z=L, t)=0 & \text { for } t>0,
\end{array}
$$

where $z=0$ at the bottom soil surface and is positive upwards, and the total height of soil in the column is L. Again, the transient behavior is neglected. The steady sinusoidal solution for the pressure at any height $\mathrm{z}$ is given by [Garbesi, 1993]

$$
P(z, t)=A\left\{B \cos \left(\omega t+z^{\prime}\right)+\frac{\cos \left(\omega t-z^{\prime}\right)}{B}-C \cos \left(\omega t+z^{\prime}-2 L^{\prime}\right)-\frac{\cos \left(\omega t-z^{\prime}+2 L^{\prime}\right)}{C}\right\}
$$

where $\mathrm{Z}^{\prime}, \mathrm{L}^{\prime}, \mathrm{A}, \mathrm{B}, \mathrm{C}$ are defined as

$$
\begin{aligned}
& z^{\prime}=\lambda z / \sqrt{2}, \\
& L^{\prime}=\lambda L / \sqrt{2},
\end{aligned}
$$




$$
\begin{gathered}
A=P_{0} /\left(e^{2 L^{\prime}}+e^{\left.-2 L^{\prime}-2 \cos \left(2 L^{\prime}\right)\right),}\right. \\
B=e^{\left(z^{\prime}-2 L^{\prime}\right)}, \\
C=e^{z^{\prime}} .
\end{gathered}
$$

Following the procedure illustrated in the previous section, (22) can be solved for the time lag between the source and detector locations [Garbesi, 1993]:

$$
\mathrm{T}=\frac{1}{\omega} \tan ^{-1}\left(\frac{\delta_{1} \sin \left(\theta_{1}\right)+\delta_{2} \sin \left(\theta_{2}\right)}{\delta_{1} \cos \left(\theta_{1}\right)+\delta_{2} \cos \left(\theta_{2}\right)}\right) .
$$

where $\delta_{1}, \delta_{2}, \theta_{1}, \theta_{2}$ are defined as:

$$
\begin{aligned}
& \theta_{1}=\tan ^{-1}\left(\frac{-B \sin \left(z^{\prime}\right)+C \sin \left(z^{\prime}-2 L^{\prime}\right)}{+B \cos \left(z^{\prime}\right)-C \cos \left(z^{\prime}-2 L^{\prime}\right)}\right), \\
& \delta_{1}=\frac{-B \sin \left(z^{\prime}\right)+C \sin \left(z^{\prime}-2 L^{\prime}\right)}{\sin \left(\delta_{1}\right)}, \\
& \theta_{2}=\tan ^{-1}\left(\frac{D \sin \left(z^{\prime}\right)-E \sin \left(z^{\prime}-2 L^{\prime}\right)}{D \cos \left(\bar{z}^{\prime}\right)-E \overline{c o s}\left(\bar{z}^{\prime}-2 L^{\prime}\right)}\right),-
\end{aligned}
$$




$$
\delta_{2}=\frac{D \sin \left(z^{\prime}\right)-E \sin \left(z^{\prime}-2 L^{\prime}\right)}{\sin \left(\delta_{2}\right)} .
$$

\section{Analysis and interpretation of DDP data}

For the DDP measurements in the field and the soil column, the time lag between the source and detector pressure signals is determined by first taking the Fast Fourier Transform (FFT) of each of the signals. The channel that best represents the driving signal frequency is readily identified from the strong peak in the power spectrum at that location. The source-detector phase difference $\left(\Delta \phi_{\mathrm{m}}\right)$ is obtained by subtracting the phases indicated by the complex output of the FFT at those channels. Assuming that the signal arrives within the first period, the measured time lag is obtained from

$$
\mathrm{T}_{\mathrm{m}}=\mathrm{P}_{\mathrm{r}} \Delta \phi_{\mathrm{m}} /(2 \pi)
$$

Visual inspection of the field data was sufficient to assure that the first peak arrived at the detector location during the first signal period. Given the source and detector probe locations, the permeability is found by determining what permeability gives a theoretical time lag equal to $T_{m}$ using equation (17) or (18) for DDP field data or equation (24) for soil column data.

Signals are analyzed for phase lag only, rather than including pressure amplitude, because the signal amplitudes at the detector probes are very small $\sim \mathrm{Pa}$. Calibrating pressure transducers accurately at these low pressures is difficult, especially under field conditions. Small calibrations errors could cause large errors in the estimated permeability. In contrast, differential time lags among the transducers are easily checked in the field before and after DDP measurements, to correct for erroneous contributions to the time lag. We have found these calibrations to be quite stable under field conditions. 
Model of steady-pressure measurements of permeability in situ

Analysis of the SSP measurements uses Darcy's law and the geometry of the probe. An equivalent homogeneous permeability is determined from

$$
k=\mu Q /\left(S \Delta P_{f}\right) \text {, }
$$

where $\Delta \mathrm{P}_{\mathrm{f}}$ is the disturbance pressure difference between the soil surface and the probe tip, and $\mathrm{S}$ is a shape factor, which depends on the geometry of the probe. For the blunt-end probe, modeled as a spherical pressure source, $S=4 \pi \mathrm{r}$, where $\mathrm{r}$ is the probe radius ([Garbesi, 1988], Appendix), Numerical modeling indicates that $S=0.3 \mathrm{~m}$ for the well-screen probes [Fisk et al., 1992].

As discussed in the introduction, the effective sampling radius (or sampling scale) of these types of measurements is taken as the radial distance within which the pressure field is greater than or equal to $5 \%$ of its value at the probe tip. Using this criterion, the sampling scale for blunt-end probe is $10 \mathrm{~cm}$, whereas the sampling scale for the well-screen probe is $50 \mathrm{~cm}$ [Fisk et al., 1992].

Model of steady -pressure measurements of permeability in the soil column:

The original one-dimensional version of Darcy's law is used to determine the integrated permeability between any two ports in the soil column. Solving for the permeability yields

$$
\mathrm{k}=\mu \mathrm{QL}_{\mathrm{s}} /\left(\mathrm{A}_{\mathrm{c}} \Delta \mathrm{P}_{\mathrm{s}}\right) \text {, }
$$

where $L_{s}$ is the distance between the two ports across which a pressure difference, $\Delta P_{s}$, is measured, and $A_{c}$ is the cross-sectional area of the soil column. 


\section{MEASUREMENTS}

\section{Field Measurements of Permeability}

An intensive field investigation was carried out at one site (BLS92), to verify the DDP measurement technique and to study the soil for scale dependence of permeability. Given the strong scale dependence found at this site, the effect was studied at two additional sites (ANAS and BLF94) to determine if the result was an anomalous feature of the first site, or if it is also present in other rather different soils. The local topography at all sites was fairly flat. Table 1 indicates the site-specific parameters used in the analysis of the DDP data.

The soils and histories of the three sites are very different. BLS92 consists of a residual sandy-loam soil, of granitic parent material, weathered in place [Fisk et al., 1989]. This site is located within a grove of mature Coast Live Oak trees (Quercus agrifolia) in Ben Lomond, California, and contains two experimental basements used to study radon transport through soils and into houses [Fisk et al., 1989; Fisk et al., 1992; Garbesi et al., 1993]. Field tests were carried out there in May and September of 1992, a time during which soil conditions were fairly stable [Garbesi et al., 1993]. Extensive geological and pedological characterization of the BLS92 site has been carried out in association with other projects [Brimhall and Lewis, 1992; Flexser et al., 1993].

Figure 5 shows a plan view of the BLS92 site indicating the locations of DDP and SSP measurements and the test structures. DDP measurements were made from two source probes located at depths of 1.5- and 2-m, on the south and east sides of the west structure, respectively. SSP measurements were made using both the smaller blunt-end probes and cylindrical probes indicated in the figure. DDP measurements are indicated by the arrows.

The second site (ANAS) was a closed truck-refueling station at the Alameda Naval Air Station, Alameda, California. The station was closed because of a serious fuel spill and was being studied for soil-gas transport of volatile organic contaminants. The soil at the site was $>90 \%$ sand vegetated only with sparse dry grass. SSP measurements were made at 11 probes. DDP measurements were carried out from June to July, 1994. All DDP measurements were made from a single source located at $1.0-\mathrm{m}$ depth. The $16 \mathrm{DDP}$-detector probes were located at distances between 0.5 and $4 \mathrm{~m}$ from the source probe, primarily in one horizontal plane at the source probe depth. To explore anisotropy, DDP measurements between the source and three detector probes located $1 \mathrm{~m}$ from source probe and at a $45^{\circ}$ angle (Figure 4). In plan view, measurements were made along three rays spaced $90^{\circ}$ apart. 
BLF94 is a fallow silvicultural field located adjacent to the BLS92 site. The soil and vegetation at BLF94 was historically the same as the BLS92 site, but has been significantly amended and disturbed over many years for the cultivation of tree seedlings. The date of forest clearing is unknown, but regular cultivation began in the late $1960 \mathrm{~s}$. The soils are prepared for sowing by chiseling down to $\sim 0.5 \mathrm{~m}$. The soil is then tilled and worked with mushroom compost to a depth of $\sim 0.2 \mathrm{~m}$. Sowing occurred at intervals between 1 and 4 years, with individual crops typically harvested within a year of planting $(\mathrm{H}$. Wells, Director, Ben Lomond Nursery, personal interview, 1994). DDP measurements were made in August 1994 from a single source located at $1.5 \mathrm{~m}$ depth to 16 detector probes located between 0.5 and $4.5 \mathrm{~m}$ from the source. Measurement paths were primarily in one horizontal plane at the source probe depth. To explore anisotropy, DDP measurements were between the source and three detector probes located $1.5 \mathrm{~m}$ from source probe and at a $45^{\circ}$ angle (Figure 4). In plan view, measurements were made along three rays spaced $120^{\circ}$ apart. SSP measurements were not made at this site because of time constraints.

\section{RESULTS AND DISCUSSION}

\section{Field measurements}

Figures $6 a-6 c$ show the results of the permeability measurements made over different length scales at the three study sites. Because horizontal-vertical anisotropy was detected at all sites, to avoid confusing anisotropy with the scale effect, DDP data are presented only for horizontal path measurements. SSP measurements were made only at the BLS92 and ANAS sites.

The symbols in Figures $6 \mathrm{a}-6 \mathrm{c}$ represent the uncertainty-weighted geometric mean (GM) value of permeability measurements made at $\mathrm{n}$ different probes (SSP), or along $\mathrm{n}$ different paths (DDP), at a given length scale. If multiple measurements were made at the same probe or path, those values were averaged arithmetically before being used as input to the GM. The vertical bars on the SSP symbols indicate the GM times (top of bar) or divided by (bottom of bar) the geometric standard deviation (GSD); where the GSD is the standard deviation of the natural log of the permeability. Because of the relatively small number of DDP measurements at any given length scale, the vertical bars on the DDP symbols indicate the full range of values measured. For characterizing the data, the GM rather than the arithmetic mean is used because spatially-distributed permeability data are often lognormally distributed [de Marsily, 1986]. We confirmed that a lognormal distribution provides a reasonably good fit to the BLS92 SSP data set, for which sufficient data are available at a given scale to test the nature of the distribution. 
The first important result, indicated in Figures $6 \mathrm{a}$ and $6 \mathrm{~b}$, is that the SSP data support the smaller-scale DDP. The small-scale DDP measurements are validated by SSP measurements available for the BLS92 and ANAS sites (Figures $6 \mathrm{a}$ and $6 \mathrm{~b}$ ). In particular, the DDP and SSP measurements agree at the 0.5-m scale (BLS92), providing support that the DDP method accurately measures soil air-permeability in situ. The BLS92 SSP data also appear to confirm the trend of increasing permeability with measurement scale.

The second important observation is that soil permeability appears to depend on sampling scale at all three sites. At the BLS92 and ANAS sites the effect is very strong (Figures 6a and 6b). As the sampling scale increases from $0.1 \mathrm{~m}$ to $\sim 4 \mathrm{~m}$ at the BLS92 and ANAS, permeability increases by factors of 15 and 20 , respectively. The scale effect appears less strong at the BLF94 site (Figure $6 \mathrm{c}$ ), but that site is missing data at the smallest $(0.1 \mathrm{~m})$ scale measured at the other sites. If such data were available, the magnitude of scaling might be similar at all sites. At each site, permeability increases with measurement scale at smaller scales and appears to approach a constant value at larger scales. However, the scale at which permeability appears to level off is different at the different sites.

The data from BLS 92 indicate that scale dependence of permeability is not a local anomaly, but is relatively uniformly distributed throughout the soil. This finding is indicated by the fact that the two clusters of DDP data (Figure 5) are consistent (hence the relatively smooth tend in Figure 6a). In addition, a lack of obvious regional trends in the small-scale SSP measurements suggests that the characteristics of the soil structure that produce the scale dependence effect are relatively homogeneously distributed throughout the soil. This inference is further supported by the observation of a relatively symmetric pressure field surrounding the structure during depressurization [Garbesi et al., 1993].

Visual inspection of the BLS92 soil in a 3-m deep trench cut at the site, and in the excavations for the two structures, supports a hypothesis that networks of fast flow pathsprobably from plant roots and animals burrows - create the scale-dependent permeability effect at that site. These excavations revealed a relatively uniformly-distributed network of Oak tree roots down to about $1.5-\mathrm{m}$ depth, with larger roots $(-3 \mathrm{~mm}$ diameter) distributed at approximately $20 \mathrm{~cm}$ intervals. Sparser, but still obvious root penetration continues down to $\sim 2 \mathrm{~m}$. Brimhall et al. [ 1993] argue that, in general, preferential flow paths dominate mass transport through soils, and that this is true in particular at the Ben Lomond site [Brimhall et al., 1993] where "faunal burrows and root tubules control dialational mixing connected avenues for downward transport of material". Note that the emphasis in that study was on gravitationally-driven (hence vertical), water-born flows. Thus there is no implication that horizontal flows are not similarly enhanced. 
The hypothesis that preferential flow paths cause the scale-dependence effect is consistent with the data in Figures $6 \mathrm{a}$ and $6 \mathrm{~b}$. Permeability appears to be increasing up to higher scales in the BLS92 site (which contains large tree roots and evidence of gopher activity) than in either the ANAS grassy site or the BLF94 barren-field site (which have no obvious sources of large-scale fast paths). Likely sources of smaller-scale fast paths at the ANAS site include pathways from grass roots and soil mesofaunal burrowing). The structure of the BLF94 soil is not clear. It might contain fast paths from root-pathways from the historic Oak forest, as indicated by the finding of a large piece of root in one of our soil core samples. On the other hand, the tilled surface soil is well homogenized. If fast paths form nested hierarchical structures, as suggested by a number of researchers [Burrough, 1983a; Burrough, 1983b; Neuman, 1990], then the larger the path over which measurements are made, the greater the likelihood of intercepting larger, faster flow paths; hence the larger the effective permeability.

A surprising result indicated in Figures $6 \mathrm{a}$ and $6 \mathrm{~b}$ is that, although the increase of permeability with length scale ceases at a smaller scale $(\sim 1 \mathrm{~m})$ at the grassy ANAS site than at the treed BLS92 site $(\sim 3 \mathrm{~m})$, the total magnitude of the effect is actually larger at ANAS. We interpret this as an indication that the ANAS soil does have a similar structure of fast flow paths that creates the scale dependence effect, but that the largest features are smaller than at the other site. The large magnitude of scale dependence at the ANAS site is what is surprising. This is an important finding since it suggests that the scale dependence effect could be large in any soil and must never be ignored as a possible factor that could significantly influence advective transport of soil-gas contaminants. Note that if transport at the ANAS site were assessed in the usual manner using the typical $0.1-\mathrm{m}$ sampling-scale probes, bulk transport of contaminants into local buildings would be underestimated by a factor of 20 !

Anisotropy in permeability was also estimated for all sites based on equation (19) and Figure 4. For the BLS92 site anisotropy was calculated separately for measurements made at the 2-m scale (north side of structure) and at the 1.5 -m scale (east side of structure). Horizontal permeability exceeds vertical permeability by a factor of $\sim 2$, with the measurements at the two different scales agreeing within measurement uncertainty $(1.6 \pm 0.4$ and $1.8 \pm 0.4$, respectively, given a $20 \%$ uncertainty in individual measurements). Using the same estimation procedure the horizontal permeability exceeds the vertical permeability by a factor of $3 \pm 0.6$ at BLF9 4 , and by a factor of $4.6 \pm 1.0$ at ANAS. 


\section{Soil column verification}

The results of the steady-state and dynamic measurements of soil permeability made in the soil column are shown in Figure 7. The symbols indicate the weighted arithmetic mean (AM) value of repeated measurements, at a given length scale. Three repeated measurements were made at each length scale for the SSP measurements. One DDP measurement was made at the 0.5 - and $1.5-\mathrm{m}$ scales. Two DDP measurements were made at the 1.0 - and $1.85-\mathrm{m}$ scales, at different driving signal frequencies. The instrumental uncertainties of the underlying measurements, determined from standard propagation of error, are indicated by the vertical bars. Repeated measurements indicated that instrumental uncertainty is the largest source of uncertainty in these laboratory measurements.

Two features of the data are immediately obvious from Figure 7: (1) The DDP measurements in the soil column show no scale dependence of permeability in the homogeneous sand over the range of lengths scales in which scale dependence was observed in the field. The steady-pressure measurements concur with this. (2) There is a systematic discrepancy of $\sim 20 \%$ between the DDP and the steady-pressure permeability estimates suggesting a bias in one, the other, or both techniques.

The $20 \%$ discrepancy probably results from preferred flow occurring along the walls of the soil column. During steady-state experiments, in which flow is kept at some fixed value, slippage along the wall would result in higher flows than the soil itself would let pass, leading to an overestimate of the permeability. This effect should be larger in the steady-pressure measurements than the DDP measurements, in which average flows are about zero and the maximum flow is about the same as the steady flow used in the steady-state experiments. Whatever the cause, the $20 \%$ discrepancy is small relative to the magnitude of the scale-dependence effect observed in the field and therefore does not significantly alter our findings.

Two results, in particular, from the soil column experiments give us confidence in the DDP field measurements. First is the null result: that no scale dependence was observed in a system in which it is not expected, a soil column filled with uniform dry sand. In particular, no scale dependence was observed in this system over the same range of length scales in which large scale dependence of permeability was observed in the field. Second is the finding that measurements nun at different driving signal frequencies yield the same estimates of soil permeability. DDP measurements at the 1.85 -m scale using 30 -s and 120 -s driving-signal periods produced identical estimates of permeability $\left(5.6 \pm 0.3 \times 10^{-11} \mathrm{~m}^{2}\right)$. Measurements made at the $1.0-\mathrm{m}$ scale at 30 - and 60 -s periods agreed to within $2 \%$. 


\section{Other Evidence}

The large-scale DDP measurements of permeability at the BLS92 site are consistent with measurements of soil-gas entry into the Ben Lomond west structure reported earlier [Garbesi, 1993; Garbesi et al., 1993]. When the 0.5-m scale SSP measurements of permeability were used as input to a 3-dimensional finite-difference model of soil-gas entry, the model underpredicted the measured entry rate by about a factor of 8 [Garbesi et al., 1993]. Measurements of the static soilgas pressure field created by structure depressurization indicate that the structure interacts with the soil at a scale of about 3 meters [Garbesi, 1993; Garbesi et al., 1993]. When the $3-\mathrm{m}$-scale estimates of permeability are used, the finite difference model only underpredicts the measured entry rate by a factor of 1.5 [Garbesi et al., 1993; Robinson and Sextro, In Press].

Another set of experiments carried out at the BLS92 site suggests that the 3-m characterization of soil permeability is an accurate representation of the soil as "seen" by the structure, and that the remaining factor of 1.5 discrepancy in soil-gas entry rate is due to other causes: probably due to the presence of fast flow at the soil-structure interface [Garbesi, 1993]. Those measurements provided independent verification of the several-meter-scale permeability at BLS92 via data on soil-gas concentrations of ${ }^{222} \mathrm{Rn}$ during long-term steady-state depressurization of the west structure [Garbesi, 1993]. During structure depressurization, air originating at the soil surface is drawn down through the soil and enters the structure through openings in the slab floor, 1.8 -m below grade (Figure 8 illustrates the geometry for a sample experiment at $20 \mathrm{~Pa}$ depressurization). If the time for soil air to travel from the surface to a soil probe is small compared to 3.82 -day half-life of ${ }^{222} \mathrm{Rn}$, then the soil-gas radon concentration will be noticeably diluted. Greater dilution indicates higher soil permeability because the soil-gas velocity is proportional to the permeability by Darcy's law.

Figure 9 shows soil-gas radon concentrations as a function of structure depressurization. Soil-gas radon was sampled at the probe location indicated in Figure 8; concentrations are normalized with respect to the concentration at no structure depressurization. Also shown are soilradon concentrations predicted for the same location by the radon-transport model discussed above. The figure shows model predictions based on the $0.5-\mathrm{m}$-scale and the 3 -m-scale permeabilities indicated in Figure 6a. The model produces good agreement with the measured radon concentrations when the $3-\mathrm{m}$ scale permeability is used, but significantly underpredicts depletion when the 0.5-m permeability is used [Garbesi, 1993; Garbesi et al., 1993]. This is strong confirmation that the structure "sees" soil permeability corresponding to the 3-m scale DDP measurement. 


\section{CONCLUSIONS}

Field and laboratory experiments presented here verify that the DDP method for measuring soil air permeability produces accurate results and substantiate the conclusion that scale dependent permeability observed in the field is real and not an artifact of the measurement technique. The DDP method opens up new possibilities for studying soil physics and advective transport of contaminants through soils. The two probe design of the DDP method makes it ideal for detecting anisotropy of soil permeability. Future extensions of the data interpretation model will allow us to better quantify this effect, and to produce more accurate measurements in highly anistropic soils. Because of the important implications of scale dependent permeability for understanding and predicting contaminant transport through soils, more study is clearly warranted on the causes, prevalence, and magnitude of this effect in other soils. Future work is justified to examine the consistency between the scale effect reported here with the scale effects generated by ghierarchical models and by stochastic models utilizing the concept of correlation length scale.

A virtue of the DDP technique is that it yields a meaningful measure of the integrated bulk permeability of soil for scales up to many meters, given a limited number of measurements. In the case of radon entry into houses (and in most other practical applications) one is not interested in understanding the full details of soil structure that control advection. One simply requires an integrated measure of the behavior of the soil. Where decisions about remediation or siting must be made in a limited amount of time given fiscal constraints, one may be satisfied with an accurate upper level estimate of permeability, which could be used, for example, to determine maximum indoor exposures to soil-gas contaminants. Even if it were possible to characterize bulk flow based on small-scale permeability measurements, given the enormous amount of data required just to characterize a known, statistically well-behaved system [Russo and Jury, 1987b; Russo and Jury, 1987a], the data and labor required for detailed characterization are fiscally infeasible for most non-research applications.

To our knowledge, this study is the first intensive investigation of the scale dependence of soil permeability to air and the only study (in soils, aquifers, or fractured rock systems) to measure permeabilities over a significant range of scales using the same method. Although the DDP method is more costly to assemble and requires more sophistication in its use than steady-pressure techniques, this study has demonstrated that typical estimates of soil bulk permeability based on multiple small-scale measurements can produce large underestimates of gas-phase transport of contaminants through soils in field-scale systems. If the approximate scale at which a system operates is known or can be measured, then the soil permeability should be probed at that scale. If the system of interest operates over a range of length scales, then an accurate prediction of the 
advective transport of soil-gas contaminants Jequires knowledge of effective soil-permeability over that scale range and a contaminant transport model that accounts for the scale effect.

Unfortunately, there is little agreement in the literaure on the cause of scale dependent permeability or how it should be modeled (either with bierarchical or slochastic models). An interesting possibility is to attempt to capture the effect on the system by meastiring the scale dependence in the field and using that as an input to a model, rather than explicitly simulating the structures that cause the effect or assuming the nature of a stochastic field. Regardless of the cause, our finding of significant scale-dependent permeability at the three study sites-including the sandy-soil ANAS site where we did not expect to see a strong effect-argues that the possibility of scale dependence must not be negiected when assessing advective transport of soitgas contaminants at any site. 


\section{ACKNOWLEDGMENTS}

The authors thank A. Baehr, W. Fisk, A. Gadgil, C. Doughty, and Y. Tsang and the anonymous referees for Water Resources Research for valuable reviews of draft manuscripts. K. Garbesi thanks the following organizations for fellowship support: the Association of Western Universities, the Switzer Foundation, and the US Department of Energy (DOE) for an Alexander Hollaender Postdoctoral Fellowship. Support for W. Nazaroff's effort was provided by National Science Foundation Grant BCS-9057298. This work was supported by the Kearney Foundation for Soil Science and by the Director, Office of Energy Research, Office of Health and Environmental Research, Environmental Sciences Division, and by the Assistant Secretary for Conservation and Renewable Energy, Office of Building Technologies, Building Systems and Materials Division of the DOE, under Contract DE-AC03-76SF00098. 


\section{NOTATION}

\section{Roman letters}

A

$A_{j}$

b

B

C

d

$\Delta \mathrm{P}$

$\mathrm{Dp}$

$\Delta \mathrm{P}_{\mathrm{f}}$

$\Delta \mathrm{P}_{\mathrm{s}}$

h

j index for image sources.

k permeability, $\mathrm{m}^{2}$.

k45

$\mathrm{kh}$

kv

$\mathrm{k}_{\mathrm{X}}$

ky parameter used in soil-column solution (Eq. 23c).

amplitude of detector due to the jth image.

effective radius of source probe, $m$.

parameter used in soil-column solution (Eq. 23d).

parameter used in soil-column solution (Eq. 23e).

vertical distance between detector probe and soil surface, $m$.

disturbance pressure, $\mathrm{Pa}$.

pressure diffusivity $\mathrm{D}_{\mathrm{p}}=\mathrm{kPa}_{\mathrm{a}} /(\varepsilon \mu), \mathrm{m}^{2} / \mathrm{s}$.

disturbance pressure difference between probe tip and soil surface, $\mathrm{Pa}$.

disturbance pressure difference between two ports of soil column, $\mathrm{Pa}$.

horizontal distance between source and detector probes, $\mathrm{m}$.

soil permeability along ray at $45^{\circ}$ angle up from the horizontal, $\mathrm{m}^{2}$.

horizontal soil permeability $\mathrm{m}^{2}$.

vertical soil permeability, $\mathrm{m}^{2}$.

soil permeability in $\mathrm{x}$-direction $\mathrm{m}^{2}$.

soil permeability in the $\mathrm{y}$-direction, $\mathrm{m}^{2}$. 


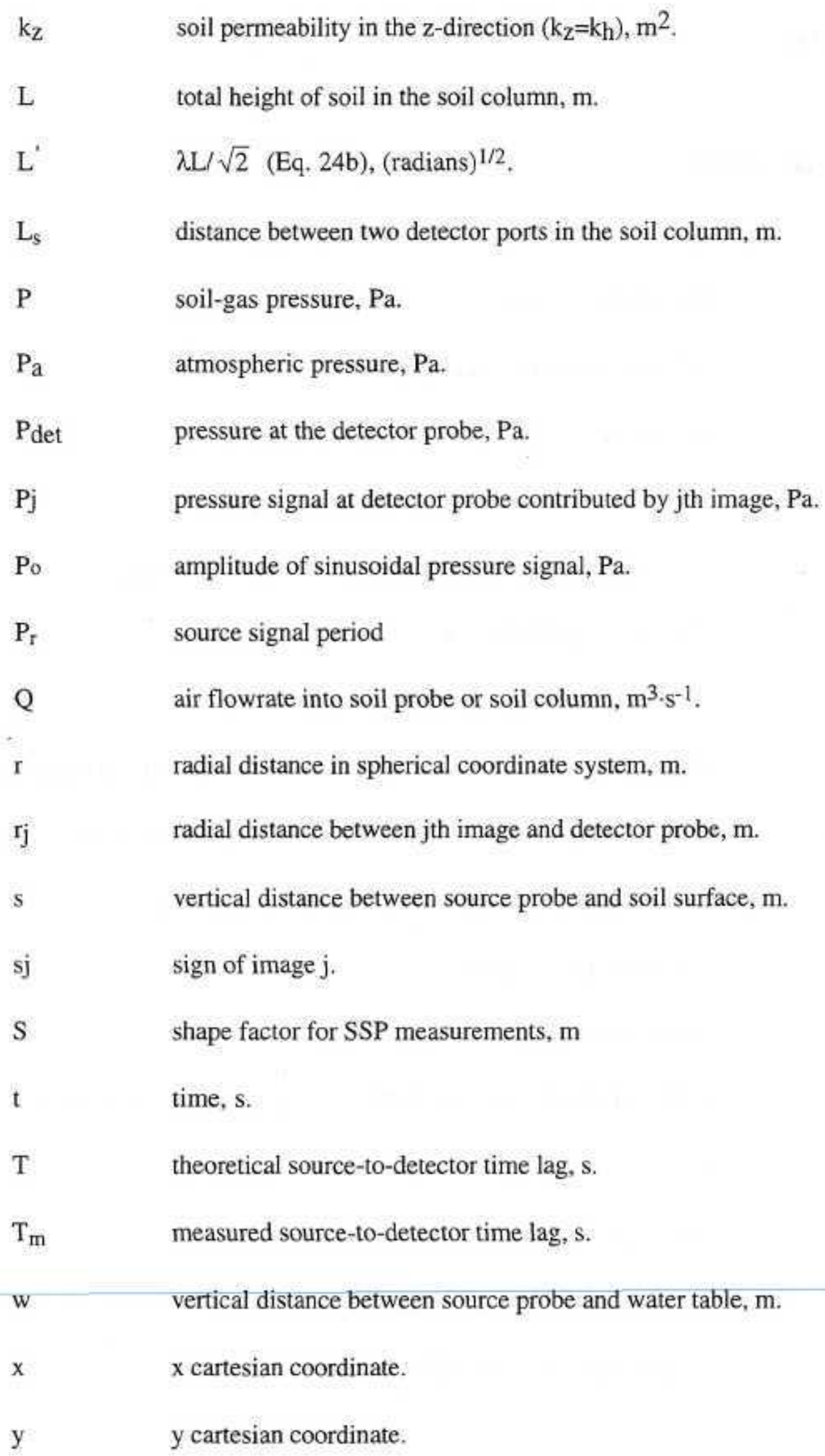




\section{Greek letters}

$\beta \quad$ phase lag of signal at detector probe, radians.

$\delta_{1} \quad$ parameter used in soil column solution (Eq. 25b)

$\delta_{2}$ parameter used in soil column solution (Eq. 25d)

$\varepsilon \quad$ air-filled porosity of soil

$\phi_{\mathrm{j}} \quad$ phase lag between detector and source due to jth image, radians.

$\Delta \phi_{\mathrm{m}} \quad$ measured phase difference between the source and detector probe, radians.

$\gamma \quad$ amplitude of signal at detector probe

$\lambda \quad$ parameter computed by $\lambda=\sqrt{\frac{\omega}{D_{p}}}, 1 / \mathrm{m}$.

$\mu \quad$ dynamic viscosity of soil gas, $\mathrm{kg} /(\mathrm{m} \mathrm{s})$.

$\theta_{1} \quad$ parameter used in soil column solution (Eq. 25a)

$\theta_{2}$ parameter used in soil column solution (Eq. 25c)

$\omega \quad$ angular frequecy of DDP source signal $\left(\omega=2 \pi / \mathrm{P}_{\mathrm{r}}\right), 1 / \mathrm{s}$. 


\section{REFERENCES}

Andersen, C. E., Entry of Soil-Gas and Radon into Houses, Rep. Riso-R-623(EN), Riso National Laboratory, Roskilde, Denmark, 1992.

Andersen, C. E., J. Sorgaard-Hansen, A. Damkjaer and B. Majborn (1995). Enhanced soil-gas entry into a radon test structure located on clayey till. Sixth International Symposium on the Natural Radiation Environment, June 5 - 9, Montreal, Canada,

Baehr, A. and M. F. Hult, Evaluation of unsaturated zone air permeability through pneumatic tests. Water Resources Research, 27(10), 2605-2617, 1991.

Baehr, A. L., G. E. Hoag and M. C. Marley, Removing volatile contaminants from the unsaturated zone by inducing advective air-phase transport. Journal of Contaminant Hydrology, 4, 1-26, 1989.

Baehr, A. L. and M. F. Hult (1989). Determination of the air-phase permeability tensor of an unsaturated zone at the Bemidji. Minnesota, research site. U.S Geological Survey Toxic Substances Hydrology Program-Proceedings of the Technical Meeting, Phoenix, Arizona, September 26-30, 1988, U.S. Geological Survey.

Baehr, A. L. and C. J. Joss, An updated model of induced airflow in the unsaturated zone. Water Resources Research, 31(2), 417-421, 1995.

Bear, J., Dynamics of Fluids in Porous Media, Dover Publications Inc., New York, 1972.

Black, J. H. and K. L. J. Kipp, Determination of hydrogeological parameters using sinusoidal pressure tests: A theoretical appraisal. Water Resources Research, 17(3), 686-692, 1981.

Blake, G. R. and K. H. Hartge (1986). Particle Density. Methods of Soil Analysis. Part I. 2nd ed. Madison, WI, American Society of Agronomy. C. A. Black, ed., 363-375. 
Bradbury, K. R. and M. A. Muldoon (1990). Hydraulic Conductivity Determinations in Unlithified Glacial and Fluvial Materials. Ground Water and Vadose Zone Monitoring. Philadelphia, American Society for Testing and Materials. D. M. Nielson and A. I. Johnson, ed., 138-151.

Brimhall, G. H., O. A. Chadwick, C. J. Lewis, W. Compston, I. S. Williams, K. J. Danti, W. E. Dietrich, M. E. Powers, D. Henricks and J. Bratt, Deformational mass transport and invasive processes in soil evolution. Science, 255, 695-702, 1993.

Brimhall, G. H. and C. J. Lewis, Differential Element Transport in the Soil Profile at the Ben Lomond Small Structure Radon Site: A Geochemical Mass Balance Study, Rep. Department of Geology and Geophysics, University of California, Berkeley, 1992.

Brimhall, G. H., C. J. Lewis, H. A. Wollenberg, S. Flexser and A. R. Smith, Soil permeability and deformational mass transport controls on radon emanation. EOS, 74(43), 299, 1993.

Burrough, P. A., Multiscale sources of spatial variation in soil. II. A non-Brownian fractal model and its application in soil survey. Journal of Soil Science, 34, 599-620, 1983a.

Burrough, P. A., Multiscale sources of spatial variation in soil. I. The application of fractal concepts to nested levels of soil variation. Journal of Soil Science, 34, 577-597, $1983 \mathrm{~b}$.

California Air Resources Board (1989). The Landfill Gas Testing Program: A Second Report to the California Legislature. Presented at a meeting of the Air Resources Board, Sacramento, CA, June 8 - 9 ,

Carslaw, H. S. and J. C. Jaeger, Conduction of Heat in Solids, Clarendon Press, Oxford, 1959.

Celia, M. A. and I. Rodrígues-Iturbe, Forward. Advances in Water Resources. Special Issue: Research Perspectives in Hydrology, 16(1), 1, 1993.

Chatfield, C., The Analysis of Time Series: An Introduction, Chapman and Hall, New York, 1989. 
Cripps, A. (1993). Gas flow rates into voids below timber floors, theory and experiment. Paper presented at AARST/EPA Conference, Denver, Colorado, Septemebr 20-23.,

Danielson, R. E. and P. L. Sutherland (1986). Porosity. Methods of Soil Analysis, Part I. 2nd ed. Madison, WI, American Society of Agronomy. C. A. Black, ed., 443-450.

de Marsily, G., Quantitative Hydrogeology, Academic Press, Inc., New York, 1986.

Fisk, W. J., S. Flexser, A. J. Gadgil, H. Y. Holman, M. P. Modera, T. N. Narasimhan, T. Nuzum, K. L. Revzan, R. G. Sextro, A. R. Smith, Y. W. Tsang and H. A. Wollenberg, Monitoring and Modeling for Radon Entry into Basements: A Status Report for the Small Structures Project, Rep. LBL-27692, Lawrence Berkeley Laboratory: Berkeley, CA, 1989.

Fisk, W. J., M. P. Modera, R. G. Sextro, K. Garbesi, H. A. Wollenberg, T. N. Narasimhan, T. Nuzum and Y. W. Tsang, Radon Entry into Basements: Approach, Experimental Structures, and Instrumentation of the Small Structures Project, Rep. LBL-31864, Lawrence Berkeley Laboratory: Berkeley, CA, 1992.

Flexser, S., H. A. Wollenberg and A. R. Smith, Distribution of radon sources and effects on radon emanation in granitic soil at Ben Lomond, California. Environmental Geology, 22, 162-177, 1993.

Freeze, R. A. and J. A. Cherry, Groundwater, Prentice-Hall, Inc,, Englewood Cliffs, New Jersey, 1979.

Fukuda, H., Air and vapor movement in soil due to wind gustiness. Soil Science, 4, 249-256, 1955.

Garbesi, K., Experiments and Modeling of the Soil-Gas Transport of Volatile Organic Compounds into a Residential Basement, MS Thesis, Rep. LBL-25519 Rev., Lawrence Berkeley Laboratory, 1988.

Garbesi, K. Toward Resolving Model-Measurement Discrepancies of Radon Entry into Houses, Ph.D. Dissertation. Lawrence Berkeley Laboratory, Report LBL-34244 (Rev.). 1993 
Garbesi, K. and R. G. Sextro, Modeling and field evidence of pressure-driven entry of soil gas into a house through permeable below-grade walls. Environmental Science and Technology, 23(12), 1481-1487, 1989.

Garbesi, K., R. G. Sextro, W. J. Fisk, M. P. Modera and K. L. Revzan, Soil-gas entry into an experimental basement: Model-measurement comparisons and seasonal effects. Environmental Science and Technology, 27, 466-473, 1993.

Hillel, D., Applications of Soil Physics, Academic Press, New York, 1980.

Hodgson, A. T., K. Garbesi, R. G. Sextro and J. M. Daisey, Soil-gas contamination and entry of volatile organic compounds into a house near a landfill. Journal of the Air and Waste Management Association, 42(3), 277-283, 1992.

Little, J. C., J. M. Daisey and W. W. Nazaroff, Transport of subsurface contaminants into buildings: An exposure pathway for volatile organics. Environmental Science and Technology, 26(11), 2058-2066, 1992.

Loureiro, C. O., L. M. Abriola, J. E. Martin and R. G. Sextro, Three-dimensional simulation of radon transport into houses with basements under constant negative pressure. Environmental Science and Technology, 24(9), 1338-1348, 1990.

Massmann, J. W., Applying groundwater flow models in vapor extraction system design. Journal of Environmental Engineering, 115(1), 129-149, 1989.

Menduni, G., Scaling permeability in fractured rocks. EOS, Transactions of the American Geophysical Union, 73(43), 214, 1992.

Muskat, M. and H. Bostet, Flow of gas through porous materials. Physics, 1, 2747, 1931.

Myers, G. E., Analytical Methods in Conduction Heat Transfer, Genium, Schenectady, NY, 1987. 
Narasimhan, T. N., B. Y. Kanehiro and P. A. Witherspoon, Interpretation of Earth Tide Responses of Three Deep, Confined Aquifers. Journal of Geophysical Research, 89(B3), 1913-1924, 1984.

National Research Council, Comparative Dosimetry of Radon in Mines and Homes, Panel on Dosimetric Assumptions Affecting the Application of Radon Risk Estimates, National Academy Press, Washington, DC, 1991.

Nazaroff, W. W., Radon Transport from Soil to Air. Reviews of Geophysics, 30(2), 137-160, 1992.

Nazaroff, W. W., S. R. Lewis, S. M. Doyle, B. A. Moed and A. V. Nero, Experiments on pollutant transport from soil into residential basements by pressure-driven airflow. Environmental Science and Technology, 21, 459-466, 1987.

Neuman, S. P., Universal scaling of hydraulic conductivities and dispersivities in geologic media. Water Resources Research, 26(8), 1749-1758, 1990.

Nielson, K. K., V. C. Rogers, V. Rogers and R. Holt, The RAETRAD model of radon generation and transport from soils into slab-ob-grade houses. Health Physics, 67(4), 363-377, 1994.

Noggle, J., Physical Chemistry, Little Brown, Boston, Mass., 1985.

Reitz, J. R., F. J. Milford and R. W. Christy, Foundations of Electromagnetic Theory, AddisonWesley, Reading, Massachusetts, 1979.

Revzan, K. L., W. J. Fisk and A. J. Gadgil, Modeling radon entry into houses with basements: Model description and verification. Indoor Air, 1(2), 173-189, 1991.

Robinson, A. L. and R. G. Sextro, The Influence of a Subslab Gravel Layer and Open Area on Soil-Gas and Radon Entry into Two Experimental Basements. Health Physics, , In Press.

Russo, D. and W. A. Jury, A theoretical study of the estimation of the correlation scale in spatially variable fields. 1. Stationary fields. Water Resources Research, 23(7), 1257-1268, 1987a. 
Russo, D. and W. A. Jury, A theoretical study of the estimation of the correlation scale in spatially variable fields. 2. Nonstationary fields. Water Resources Research, 23(7), 1269-1279, $1987 \mathrm{~b}$.

Schery, S. D. and D. Siegel, The role of channels in the transport of radon from the soil. Journal of Geophysical Research, 91(B12), 12366-12374, 1986.

Sherwood, A. E., In-situ permeability analysis using barometric pressure fluctuations, Rep. UCLR-51687, Lawrence Livermore Laboratory, 1974.

Turk, B. H., J. Harrison, R. J. Prill and R. G. Sextro, Developing soil gas and ${ }^{222}$ Rn entry potentials for substructure and assessing ${ }^{222} \mathrm{Rn}$ control diagnostic techniques. Health Physics, 59(4), 405-419, 1990.

Turk, B. H., R. J. Prill, D. T. Grimsrud, B. A. Moed and R. G. Sextro, Radon and Remedial Action in Spokane Valley Homes, Volume I: Experimental Design and Data Analysis, Rep. LBL-23430, Lawrence Berkeley Laboratory, Berkeley, CA, 1987.

Weeks, E. P., Field Determination of Vertical Permeability to Air in the Unsaturated Zone, Rep. U.S. Geological Survey Professional Paper 1051, United States Government Printing Office, Washington, DC, 1978.

Wolf, S. H., M. A. Celia and K. M. Hess, Evaluation of hydraulic conductivities calculated from multiport-permeameter measurements. Ground Water, 29(4), 516-525, 1991.

Wood, J. A. and M. L. Porter, Hazardous pollutants in class II landfills. Journal of the Air Pollution Control Association, 37, 609-615, 1987. 
Table 1. Site specific parameters used for analyzing DDP data.

\begin{tabular}{llll}
\hline Parameter & BLS92 site & ANAS site & BLF94 site \\
\hline Ground water depth ${ }^{\mathrm{a}}$ & $>14.7 \mathrm{~m}$ & $2.0 \mathrm{~m}$ & $8.0 \mathrm{~m}$ \\
air-filled porosity & $0.45^{\mathrm{b}}$ & $0.43^{\mathrm{c}}$ & $0.32 \mathrm{~d}$ \\
$\begin{array}{l}\text { (range of values) } \\
\text { mean atmospheric }\end{array}$ & $92 \mathrm{kPa}$ & $(0.36-0.47)$ & $(0.26-0.38)$ \\
pressure & $100 \mathrm{kPa}$ & $92 \mathrm{kPa}$ \\
source probe depth & 1.5 and $2 \mathrm{~m}$ & $1 \mathrm{~m}$ & $1.5 \mathrm{~m}$ \\
\hline
\end{tabular}

a. Determined from well measurements on site.

b. Calculated from the absolute porosity reported in [Brimhall et al., 1993] and from the average soil moisture content measured using a time domain reflectometer device (Trase, System 1, Soil moisture Equipment Corp., Santa Barbara, CA).

c. Determined using standard gravimetric total porosity [Danielson and Sutherland, 1986] and particle density [Blake and Hartge, 1986] measurements on 8 cores samples from two vertical profiles extending to a depth of $1.24 \mathrm{~m}$.

d. Determined as in note b, except based on 8 cores sampled from one profile at even intervals down to $2.0 \mathrm{~m}$ depth. 


\section{Figure Captions}

Figure 1. Schematic of the dual-probe dynamic pressure (DDP) apparatus for measuring soil permeability to air. The figure shows the source probe and one detector probe and the locations of pressure transducers and computer controlled three way valves ('o' indicates port that is always open).

Figure 2. Schematic of soil-column showing the locations of the signal input port, the pressure samping ports ( $\mathrm{P} 0$ to $\mathrm{P} 4$ ), and an optional cap containing flow-collector port (P5).

Figure 3. Geometry for method of images used to solve the soil surface and groundwater table boundary conditions for the DDP analysis model. The figure shows the real source and the first 5 image sources of an infinite series, and indicates the sign of each source. Also shown is a hypothetical location of a detector probe and various distances $\left(\mathrm{s}, \mathrm{w}, \mathrm{d}\right.$, and $\mathrm{r}_{\mathrm{j}}$ ) used in calculating the pressure at the detector location.

Figure 4. Configuration for measuring anisotropy of soil permeability. Two detector probes are placed equidistant from the source probe, one at the same depth as the source, the other at a $45^{\circ}$ angle $(\theta)$, with respect to the horizontal.

Figure 5. A plan view of the BLS92 site indicating the two test structures, the locations of two clusters of DDP measurements, and the locations of different types of probes used for SSP measurements. DDP measurement paths are indicated by arrows. SSP measurements were made at all probes shown in the figure. Detector probe depths ranged from 0.18 to $2.0 \mathrm{~m}$. 
Figure 6. Permeability vs. sampling length scale for measurements made in soil at the BLS92 site (a), the ANAS site (b), and the BLF94 site (c). Symbols indicate the geometric mean value of $\mathrm{n}$ measurements made at a given scale. Diamond symbols indicate DDP measurements made along horizontal paths; square symbol indicates 0.1 -m scale SSP measurements; circular symbols indicate 0.5-m scale SSP measurements. Vertical bars indicate the full range of values for DDP measurements $(n>1)$. For SSP measurements, the top of vertical bar is the GM times the GSD, the bottom of bar is the GM divided by the GSD.

Figure 7. Results of DDP measurements (square symbols) and SSP measurements (circles) made in the soil column homogeneously packed with air dry sand. The vertical bars indicate the estimated uncertainty.

Figure 8. Schematic showing a vertical cross section of the Ben Lomond west structure during a soil-radon depletion experiment with the structure held at $20 \mathrm{~Pa}$ below mean atmospheric pressure: Figure indicates the air flow from soil surface that results in dilution of soil ${ }^{222} \mathrm{Rn}$ concentrations. Also shown is the location of soil-gas radon sampling for the results indicated in Figure 9.

Figure 9. Normalized ${ }^{222} \mathrm{Rn}$ concentration vs. structure depressurization, measured at the Ben Lomond west structure at the location indicated in Figure 8. Concentrations are normalized with respect to the concentration with no depressurization. Modeled values are indicated by the curves for concentrations predicted given a soil permeability based on 0.5 -m-scale SSP measurements (dashed line) and based on 3.0-m-scale DDP measurements (solid line). 
oscillating pressure source

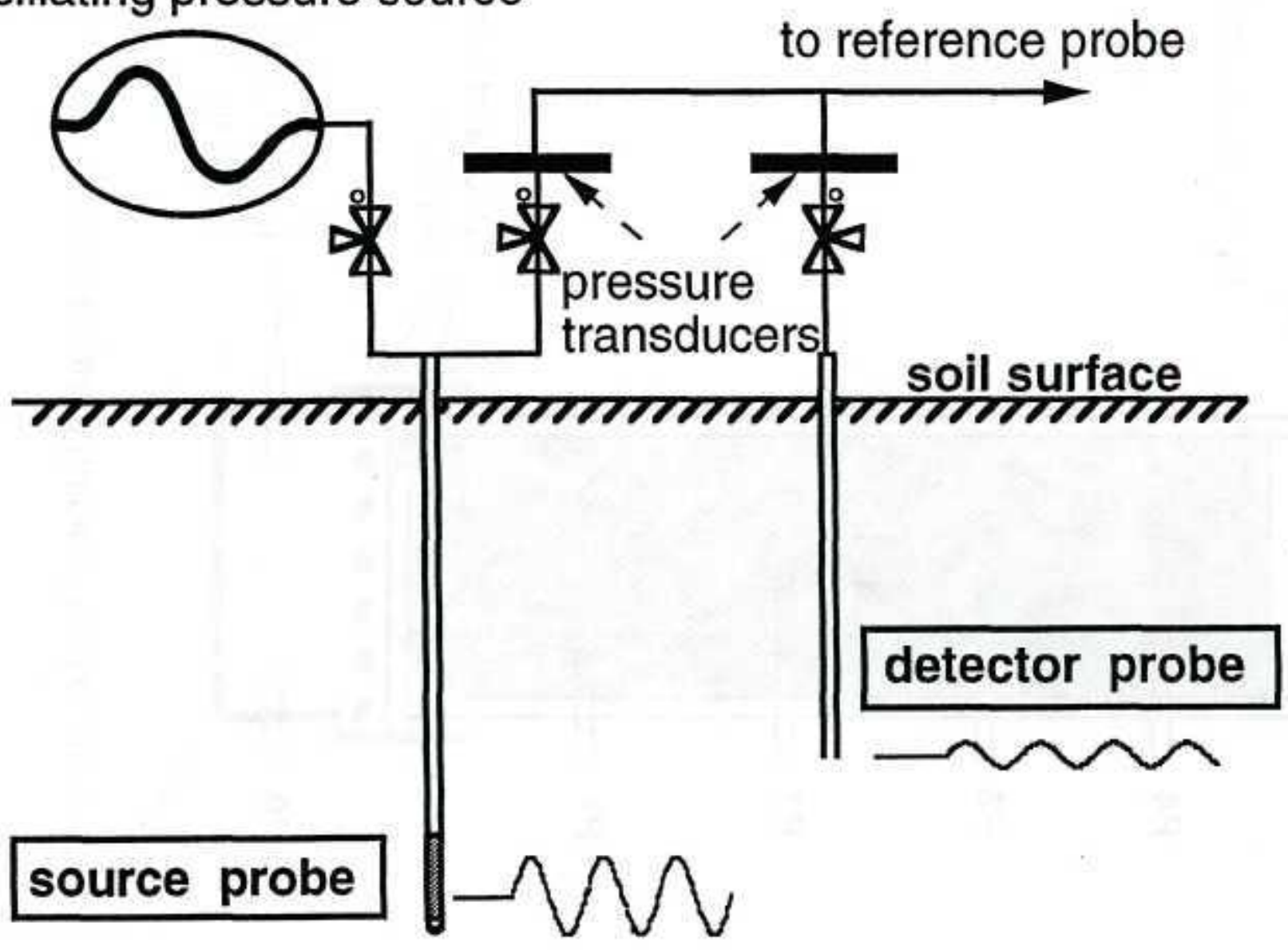


Flow collector port (P5)

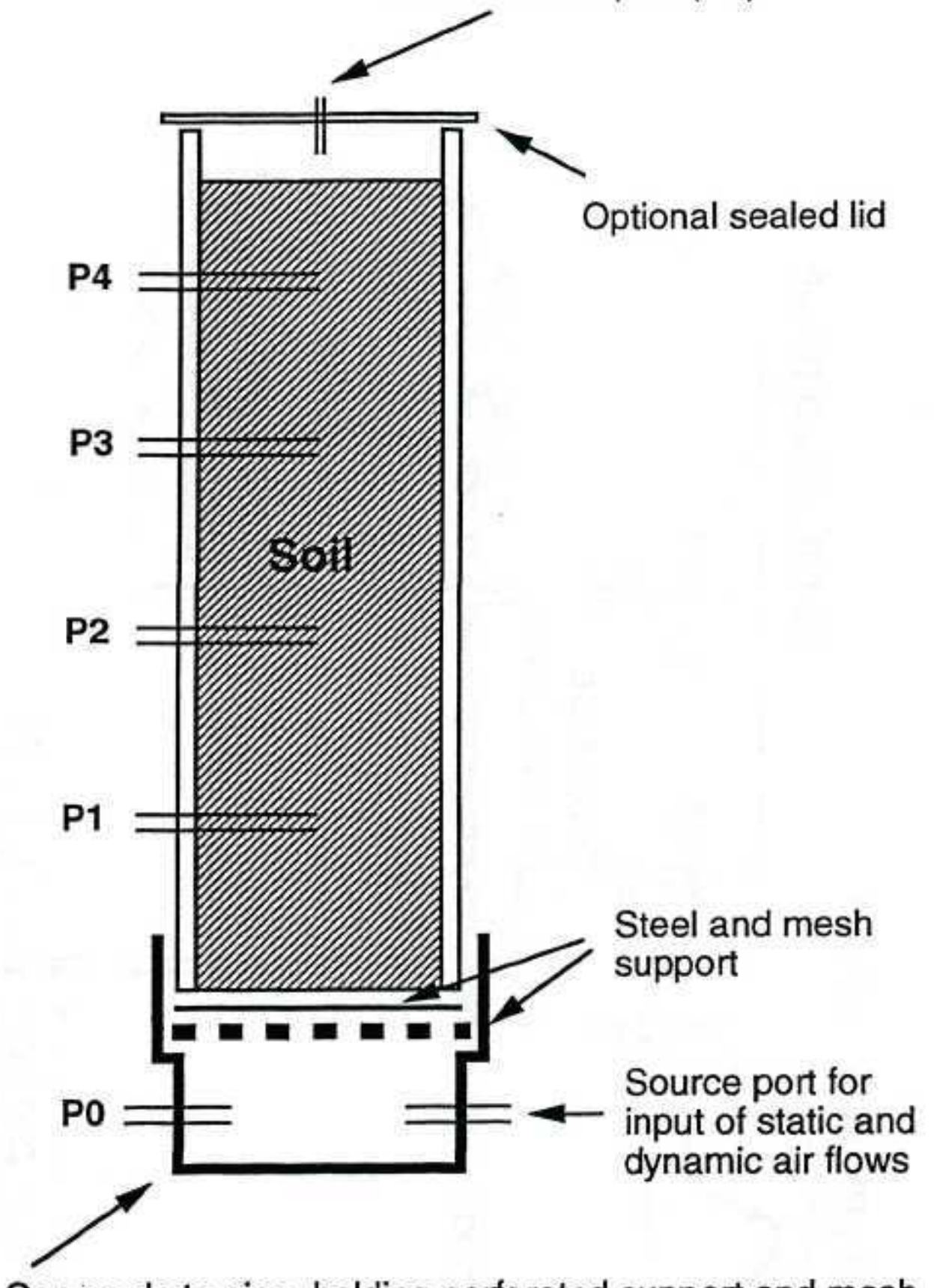

Cap seals to pipe, holding perforated support and mesh 
$2 \theta$

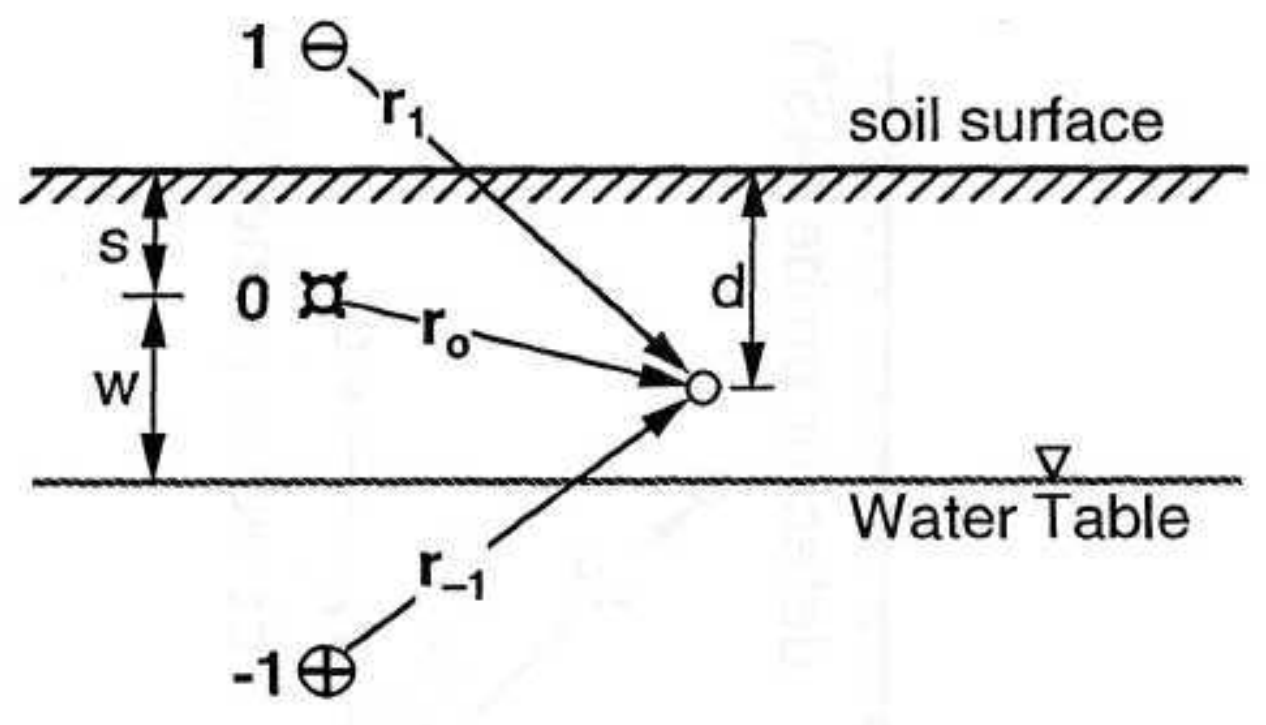

$-2 \theta$

a Real source (positive)

$\bigoplus$ Imaginary source (positve)

$\Theta$ Imaginary source (negative)

$-3 \ominus$ O Detector probe 
soil surface

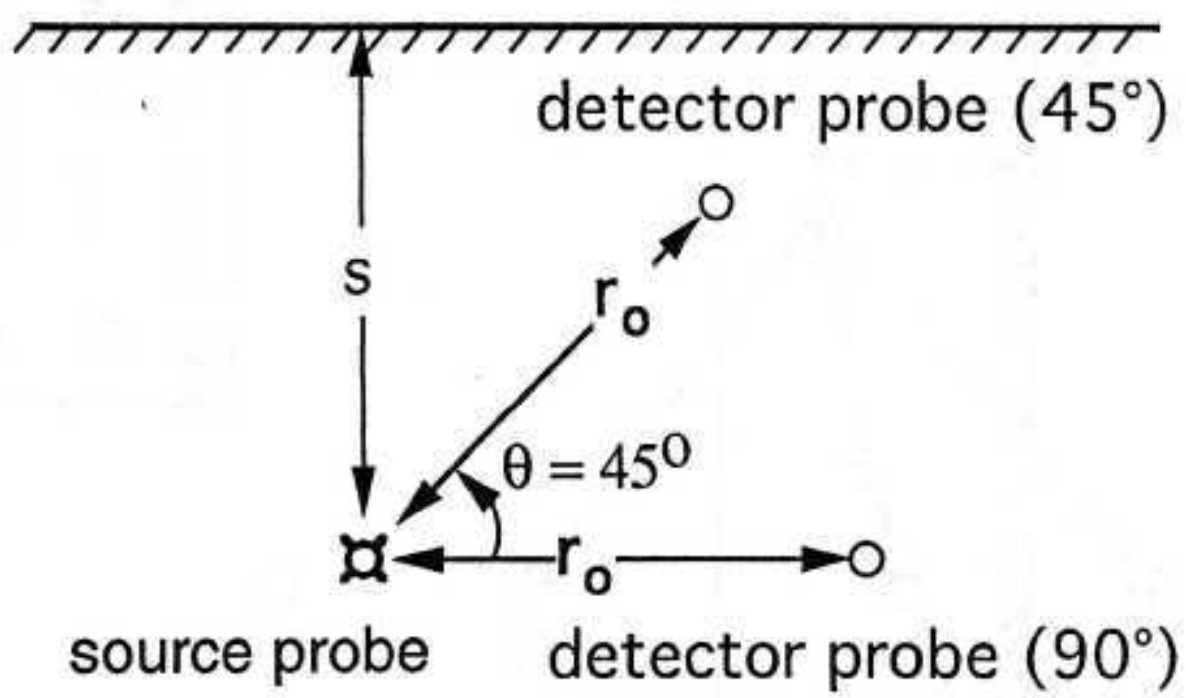




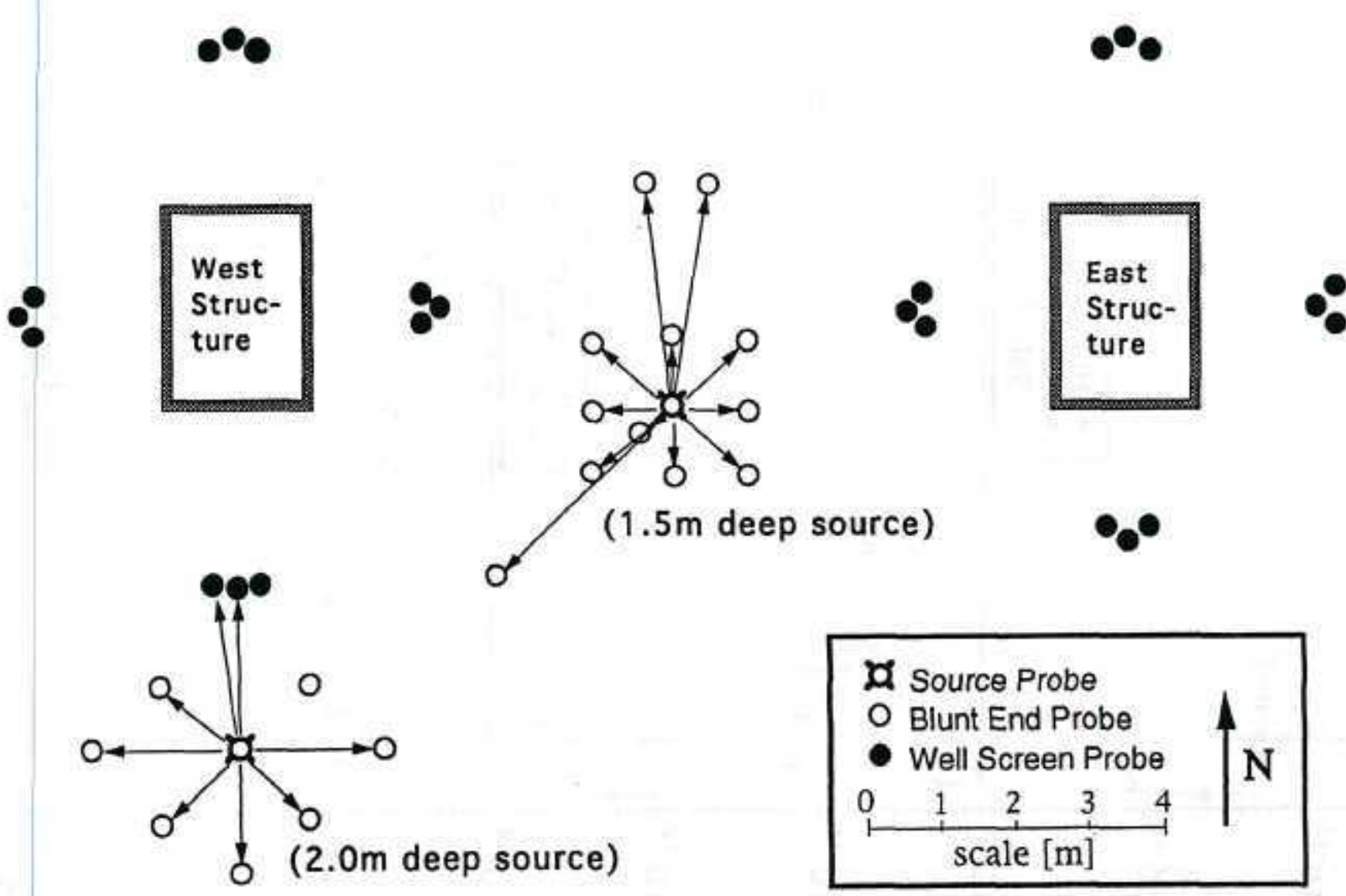




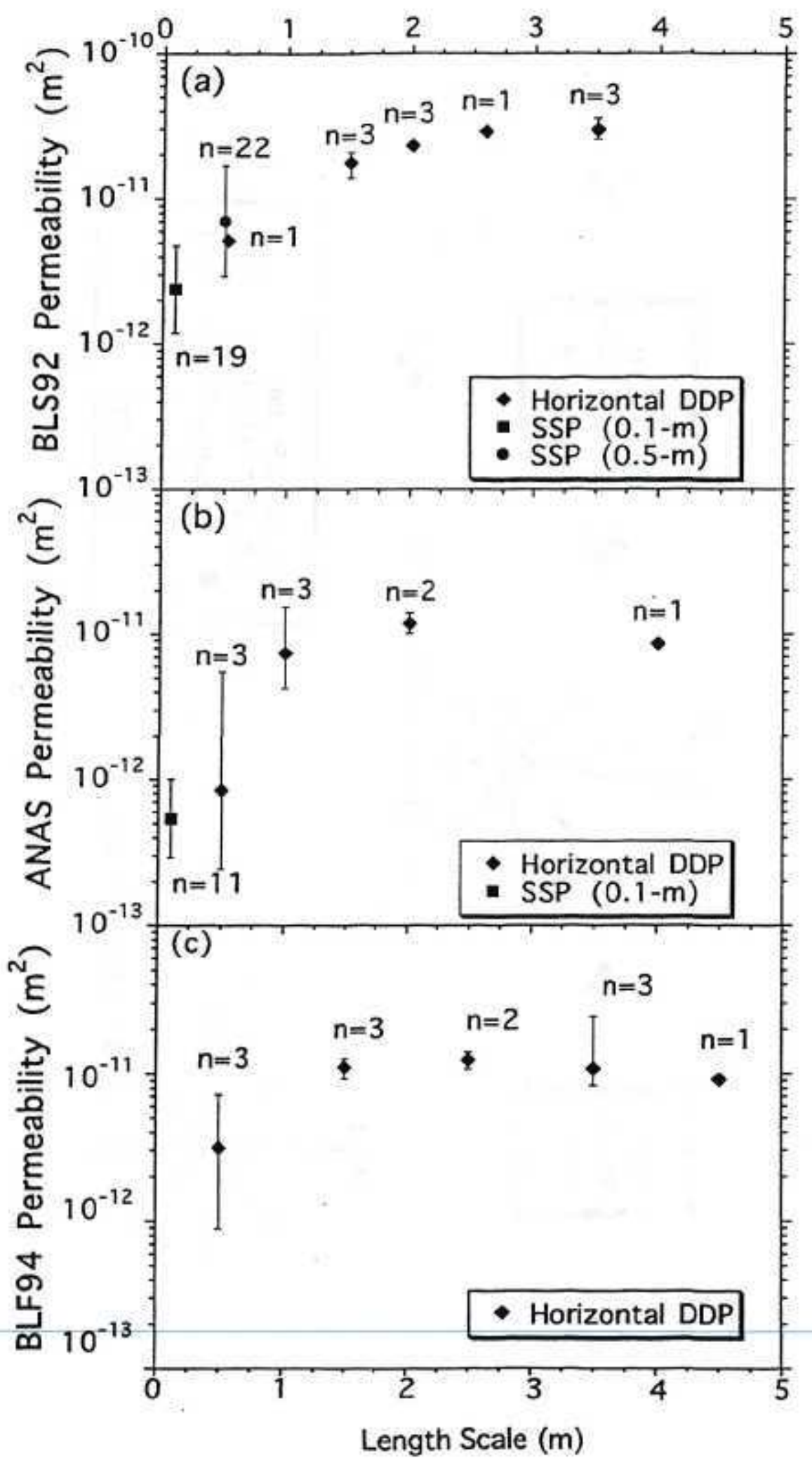




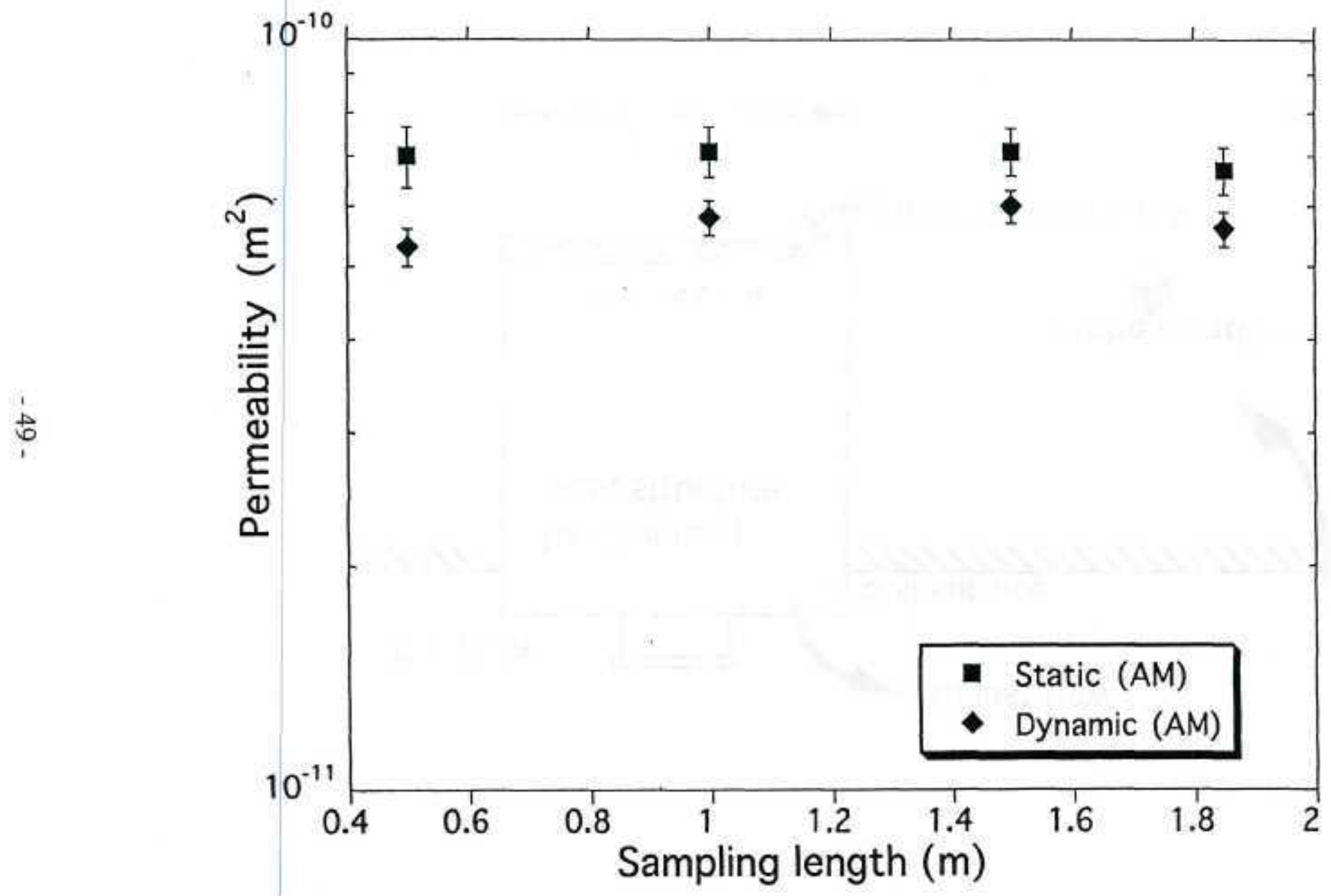




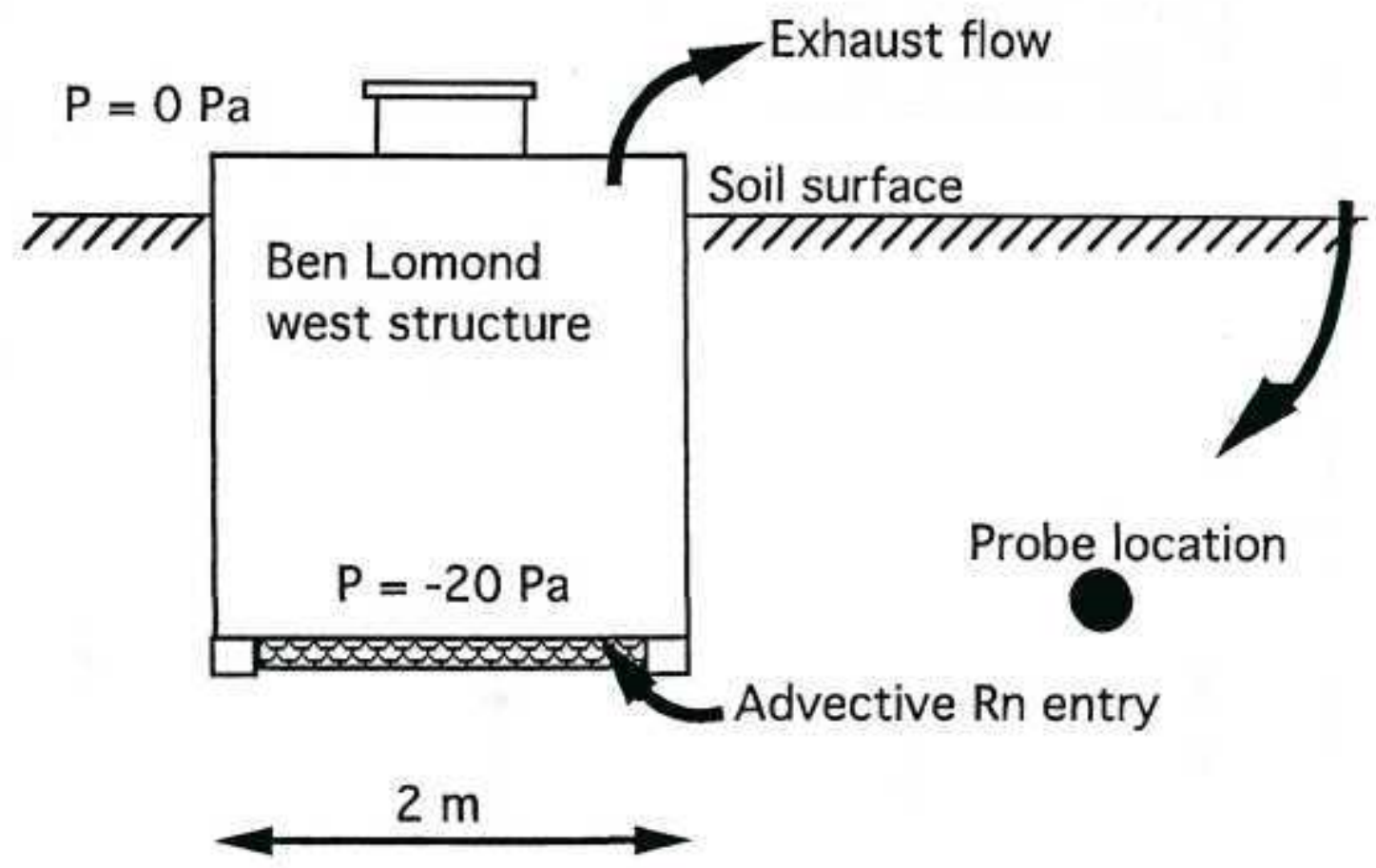




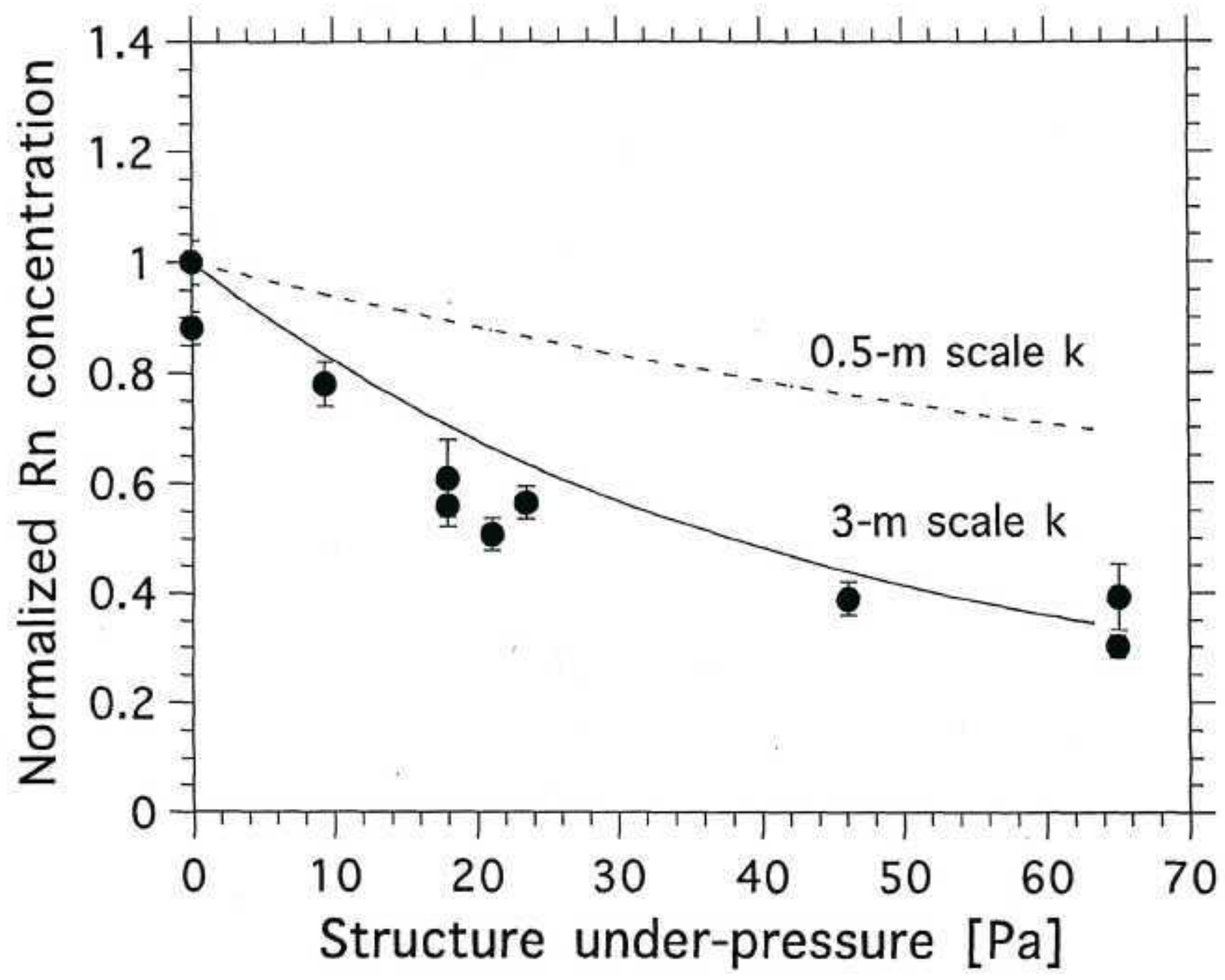





\section{DISCLAIMER}

This document was prepared as an account of work sponsored by the United States Government. While this document is believed to contain correct information, neither the United States Government nor any agency thereof, nor the Regents of the University of California, nor any of their employees, makes any warranty, express or implied, or assumes any legal responsibility for the accuracy, completeness, or usefulness of any information, apparatus, product, or process disclosed, or represents that its use would not infringe privately owned rights. Reference herein to any specific commercial product, process, or service by its trade name, trademark, manufacturer, or otherwise, does not necessarily constitute or imply its endorsement, recommendation, or favoring by the United States Government or any agency thereof, or the Regents of the University of California. The views and opinions of authors expressed herein do not necessarily state or reflect those of the United States Government or any agency thereof or the Regents of the University of California, 
